\title{
CaDHN4, a Salt and Cold Stress-Responsive Dehydrin Gene from Pepper Decreases Abscisic Acid Sensitivity in Arabidopsis
}

\author{
Hua-feng Zhang ${ }^{\dagger}$, Su-ya Liu ${ }^{\dagger}$, Ji-hui Ma, Xin-ke Wang, Saeed ul Haq, Yuan-cheng Meng, \\ Yu-meng Zhang and Ru-gang Chen * \\ College of Horticulture, Northwest A\&F University, Yangling 712100, China; 18848966687@163.com (H.-f.Z.); \\ YaSuLiu@126.com (S.-y.L.); jihuima@126.com (J.-h.M.); W1942399775@126.com (X.-k.W.); \\ saeed_ulhaq@nwafu.edu.cn (S.u.H.); YuanchengMeng07@126.com (Y.-c.M.); \\ Kexuanzhangyumeng@163.com (Y.-m.Z.) \\ * Correspondence: rugangchen@nwsuaf.edu.cn; Tel.: +86-29-8708-2613; Fax: +86-29-8708-2613 \\ t These authors contribute equally to this work.
}

Received: 30 November 2019; Accepted: 17 December 2019; Published: 19 December 2019

\begin{abstract}
Dehydrins play an important role in improving plant resistance to abiotic stresses. In this study, we isolated a dehydrin gene from pepper (Capsicum annuum L.) leaves, designated as CaDHN4. Sub-cellular localization of $\mathrm{CaDHN} 4$ was to be found in the nucleus and membrane. To authenticate the function of $\mathrm{CaDHN} 4$ in cold- and salt-stress responses and abscisic acid (ABA) sensitivity, we reduced the CaDHN4 expression using virus-induced gene silencing (VIGS), and overexpressed the CaDHN4 in Arabidopsis. We found that silencing of CaDHN4 reduced the growth of pepper seedlings and $\mathrm{CaDHN} 4$-silenced plants exhibited more serious wilting, higher electrolyte leakage, and more accumulation of ROS in the leaves compared to pTRV2:00 plants after cold stress, and lower chlorophyll contents and higher electrolyte leakage compared to pTRV2:00 plants under salt stress. However, CaDHN4-overexpressing Arabidopsis plants had higher seed germination rates and post-germination primary root growth, compared to WT plants under salt stress. In response to cold and salt stresses, the CaDHN4-overexpressed Arabidopsis exhibited lower MDA content, and lower relative electrolyte leakage compared to the WT plants. Under ABA treatments, the fresh weight and germination rates of transgenic plants were higher than WT plants. The transgenic Arabidopsis expressing a CaDHN4 promoter displayed a more intense GUS staining than the normal growth conditions under treatment with hormones including ABA, methyl jasmonate (MeJA), and salicylic acid (SA). Our results suggest that $\mathrm{CaDHN} 4$ can protect against cold and salt stresses and decrease ABA sensitivity in Arabidopsis.
\end{abstract}

Keywords: pepper; dehydrins; CaDHN4; abiotic stresses; abscisic acid (ABA) sensitivity

\section{Introduction}

Pepper (Capsicum annuum L.) is a popular vegetable that is appreciated by people from all over the world. Due to climate change, pepper plant growth and development are affected by different environmental conditions [1]. Pepper is sensitive to various stresses, such as high and low temperature, drought, salinity, heavy metals, and light, during the reproductive stage. These stresses negatively affect pepper germination, growth, loss of photosynthetic pigment, and also affect the reproductive characteristics by causing male sterility, reduced pollination and fertilization, increased premature flower and fruit drop; severe stress can also cause death in pepper plants. Our previous studies reported that the accumulation of DHNs in pepper play an important role in adaption to environmental stresses [2]. A number of studies have shown that dehydrins (DHNs) can prevent membrane lipid 
peroxidation in cells under oxidative stress, and DHNs have been widely associated with stress tolerance in plants [3-6].

Plant DHNs are highly hydrophilic proteins, belong to the second sub-family of the late embryogenesis abundant protein family (LEA), that accumulate during embryogenesis and respond to various stress conditions. Generally, these are hydrophilic proteins and contain three conserved motifs: the $\mathrm{K}, \mathrm{Y}$, and S fragments [7-9]. According to the conserved domains, DHNs are sub-divided into five categories: YnSKn, Kn, SKn, YnKn, and KnS [10-14].

Recent studies showed that DHNs have a characteristic irregular structure that can effectively resist freezing [15]. At low intracellular water potential, DHNs adsorb water molecules and act as osmotic regulators [16]. DHNs are proteins that are closely related to plant growth and development, besides their role in stress responses and distribution in different plant organs. Recently, many DHNs have been identified in plants, such as Arabidopsis thaliana [17], Citrus unshiu [18], maize [19], and Vigna radiata [20]. In potato, DHN10 accumulated in stems, tubers, young leaves, and flowers [21], similarly, in tobacco, KnS-DHNs were expressed in pollen [22]. In A. thaliana, DHNs ERD14 and LTI29 were distributed in root tips [16], while in rubber, $H b D H N 1$ and $H b D H N 2$ were expressed in leaves, stem, bark, and flowers [23].

Many studies have shown that abscisic acid (ABA) played an important role in regulating genes involved in the plant growth, development, and adaptation to environmental factors. For example, YnSKn- and YnKn-type DHNs were induced by ABA treatment. In rice, DHNs RAB21 [24] and A. thaliana DHNs RAB18, ERD14, and LTI29 were induced by ABA [16].

DHNs respond to different environmental factors [25]; in cucumber, Y3SK2 DHNs improved resistance to high temperature and cold stresses [26]. Likewise, in citrus, DHN, CuCOR19, when overexpressed in tobacco, reduced lipid peroxidation at low temperatures [27]. Under salt stress, in banana, overexpression of MusaDHN-1 improved the ability of plants to resist oxidative and salt stresses [28]. In wheat, YnSKn-type DHNs, TaDHN1, TaDHN2, and TaDHN3 were up-regulated by treatment with $200 \mathrm{mM} \mathrm{NaCl}$ [29], and in wheat, DHN WCOR410 maintained the stability of plasma membrane structure under drought stress [30], while in tomato, SbDHN1 improved drought resistance [31].

Thus, we speculate that $\mathrm{CaDHN} 4$ plays a crucial role in pepper defense under different abiotic stress conditions. This study aimed to determine the functional regulation of CaDHN4 under cold and salt stresses, and sensitivity to ABA. We performed the sub-cellular localization analysis. Furthermore, under cold- and salt-stress conditions, we characterized the CaDHN4 function through virus-induced gene silencing (VIGS) in pepper, and overexpression in Arabidopsis. This study provides valuable information regarding the function of this significant gene family in pepper and other important crops.

\section{Results}

\subsection{Sub-Cellular Localization of CaDHN4}

The physico-chemical properties of CaDHN4 genes in pepper are presented in Table S1, with which the localization of CaDHN4 in the nucleus of the cell was predicted. To confirm the sub-cellular localization of $\mathrm{CaDHN} 4$ in pepper, we constructed fusion expression vector of pBI221-CaDHN4-GFP through the fusion of the coding-sequence of CaDHN4 in-frame with GFP (Figure 1A). The fusion constructs pBI221-CaDHN4 and positive control pBI221 were transiently transformed into pepper leaf protoplasts and onion epidermal cells. By visualizing the GFP signal, we found that CaDHN4 was expressed in the nucleus and membrane of pepper protoplast cells and onion epidermal cells transfected with the CaDHN4-GFP gene fusion construct (Figure 1B,C). This GFP signal indicated that $\mathrm{CaDHN} 4$ is located in the cell nucleus and membrane (Figure 1). 


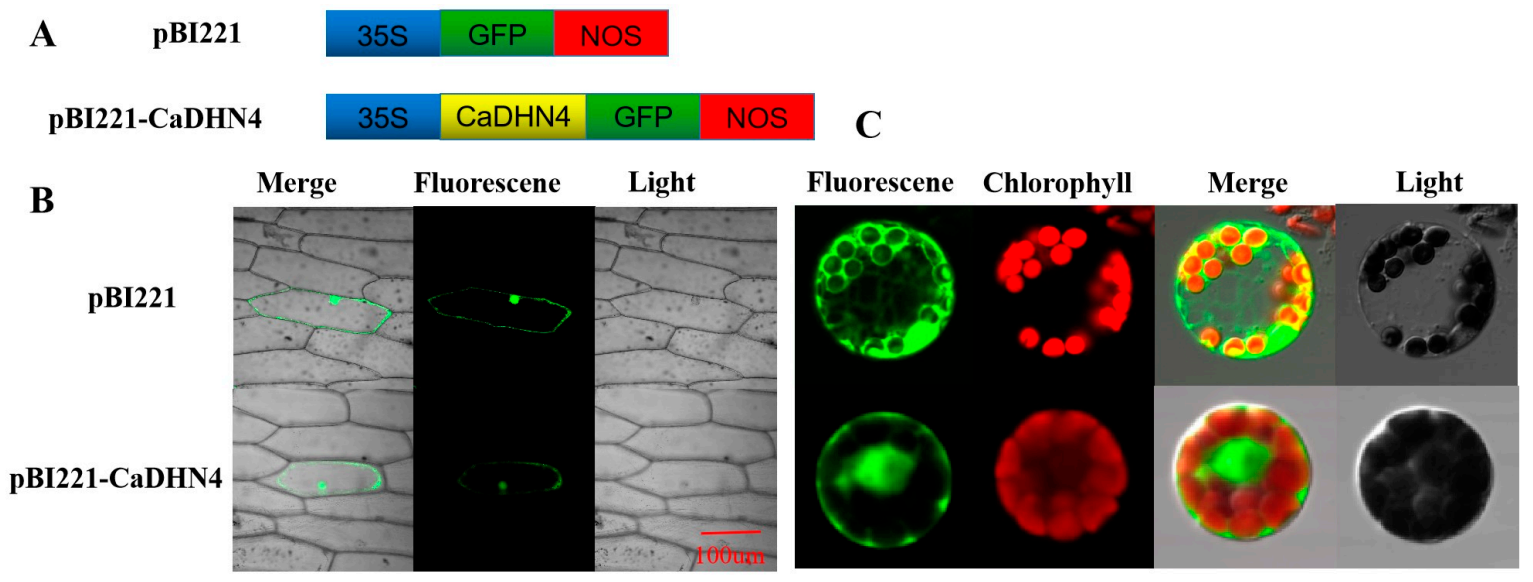

Figure 1. The sub-cellular localization of pepper CaDHN4. (A) Schematic diagram of pBI221-CaDHN4 vector. (B) Sub-cellular localization of CaDHN4 in onion epidermal cells. (C) Sub-cellular localization of $\mathrm{CaDHN} 4$ in pepper leaf protoplasts.

\subsection{GUS Histological Assay}

For GUS histological assay, $\mathrm{CaDHN} 4$ promoter region was cloned into the vector $\mathrm{pBI} 121$ to construct the plasmid CaDHN4pro:GUS (Figures 1 and 2A); the CaDHN4pro:GUS gene in transgenic plants were expressed under low temperature, salt, mannitol, drought, methyl-jasmonate (MeJA), salicylic acid (SA), and abscisic acid (ABA) hormone treatments (Figure 2B). The results indicated that CaDHN4 plays an important role in the regulation of the plant response to diverse environmental stresses.

A

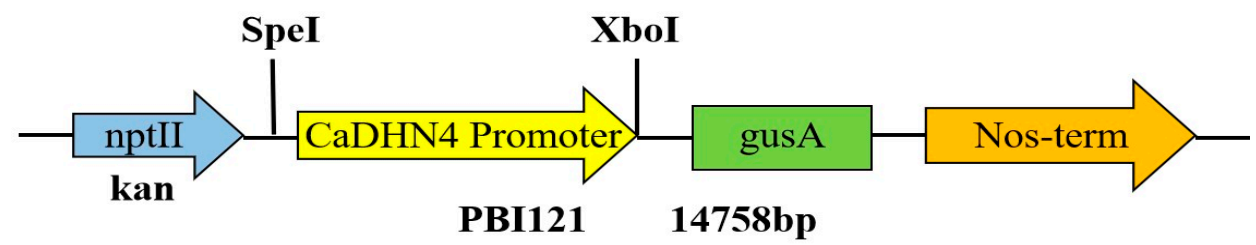

B
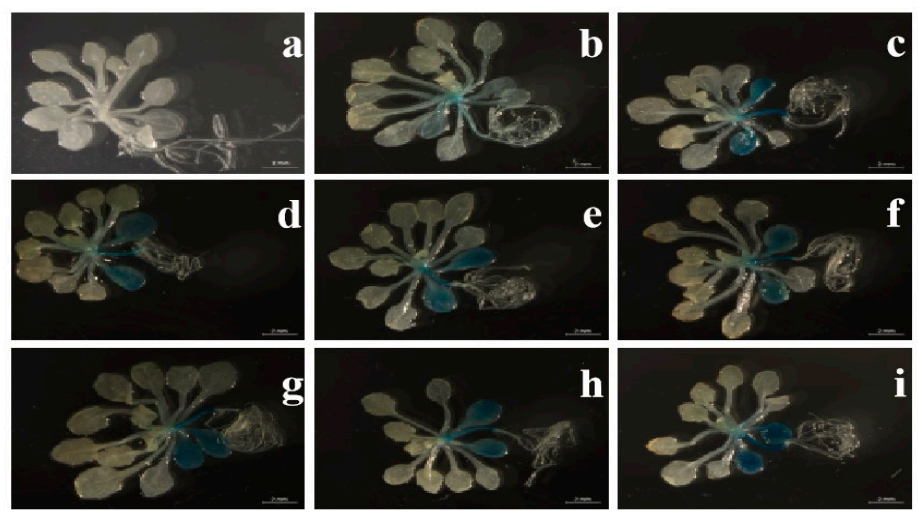

Figure 2. Analysis of CaDHN4 gene expression patterns. (A) Schematic diagram of pBI121::CaDHN4-GUS. (B) Analysis of GUS activity in transgenic Arabidopsis expressing the CaDHN4 promoter under different stress conditions. (a) Wild type; (b) transgenic Arabidopsis under normal temperature; (c) transgenic Arabidopsis under low temperature for $4{ }^{\circ} \mathrm{C}(24 \mathrm{~h}$ ); (d) $100 \mathrm{mM} \mathrm{NaCl}$ (24 h); (e) $250 \mathrm{mM}$ mannitol (24 h); (f) drought treatment (6 h); (g) $100 \mu \mathrm{M}$ methyl jasmonate (MeJA) (24 h); (h) $100 \mu \mathrm{M}$ salicylic acid (SA) (24 h); (i) $50 \mathrm{mg} / \mathrm{L}$ abscisic acid (ABA) (24 h). 


\subsection{Virus-Induced Gene Silencing (VIGS) of CaDHN4 Reduces Salt-Stress Tolerance}

To further verify the function of CaDHN4 in response to cold stress, virus-induced gene silencing (VIGS) was performed in pepper cold resistant cultivar "P70." One month later, we found that the infected pTRV2-phytoene desaturase (PDS) plants were photo-bleached (Figure S1A), which showed that the virus had successfully induced gene silencing in the pepper. The transcript levels were verified by qRT-PCR (Figure S1B). The results showed that the expression of CaDHN4 was reduced by $90 \%$, implying that $\mathrm{CaDHN} 4$ was successfully silenced through VIGS in pepper.

After 3 days' cold treatment at $4{ }^{\circ} \mathrm{C}$, the pTRV2:CaDHN4 seedlings showed more aggravated stress symptoms than pTRV2:00 plants (Figure 3A). By analyzing the relative expression of CaDHN4, we found that the expression in pTRV2:00 plants increased by almost six-folds, compared to the control plants after $24 \mathrm{~h}$ of low temperature treatment. However, the expression of pTRV2:CaDHN4 plants were only increased by four-fold, where the expression in pTRV2:CaDHN4 plants were lower than pTRV2:00 plants (Figure 3D). The relative electrolyte leakage and total chlorophyll content of pTRV2:00 and pTRV2:CaDHN4 plant leaves under cold stress were observed. The relative electrolyte leakage was higher $(\sim 15 \%)$ in pTRV2:CaDHN4 plants as compared to pTRV2:00 plants (Figure 3E), and the total chlorophyll content of pTRV2:00 plants was higher $(\sim 30 \%)$ than that of pTRV2:CaDHN4 plants (Figure 3F).

To determine the oxidative burst in pTRV2:00 and pTRV2:CaDHN4 plants, after cold stress, the contents of $\mathrm{H}_{2} \mathrm{O}_{2}$ and $\left(\mathrm{O}_{2}^{-}\right)$were investigated using a histochemical staining method with 3,3'-diaminobenzidine (DAB) and nitro-blue tetrazolium (NBT), respectively. We found an intense staining in the pTRV2:CaDHN4 leaves as compared to the pTRV2:00 plants (Figure 3B,C), which showed that reactive oxygen species (ROS) production $\left(\mathrm{H}_{2} \mathrm{O}_{2}\right.$ and $\left.\mathrm{O}_{2}{ }^{-}\right)$were higher in pTRV2:CaDHN4 plants than the pTRV2:00 plants, in response to cold stress.

Next, we measured the activities of superoxide dismutase (SOD), peroxidase (POD), catalase (CAT), and ascorbate peroxidase (APX) in the pTRV2:CaDHN4 plants and the pTRV2:00 plants under normal or cold stress conditions. In normal condition, the activity of SOD, POD, CAT, and APX were not significantly different in the control and silenced plants. When subjected to cold stress, a remarkable increase in the activities of SOD, POD, CAT, and APX were observed in the pTRV2:CaDHN4 plants and pTRV2:00 plants. However, these increases of enzymatic activities were higher in pTRV2:00 plants as compared to the pTRV2:CaDHN4 plants (Figure 3G-J). To know the ROS scavenging mechanism, we tested the accumulation of $\mathrm{H}_{2} \mathrm{O}_{2}$ in the leaves. The results showed that under cold stress, the pTRV2:CaDHN4 plants had higher $\mathrm{H}_{2} \mathrm{O}_{2}$ contents as compared to the pTRV2:00 plants (Figure $3 \mathrm{~K}$ ). 

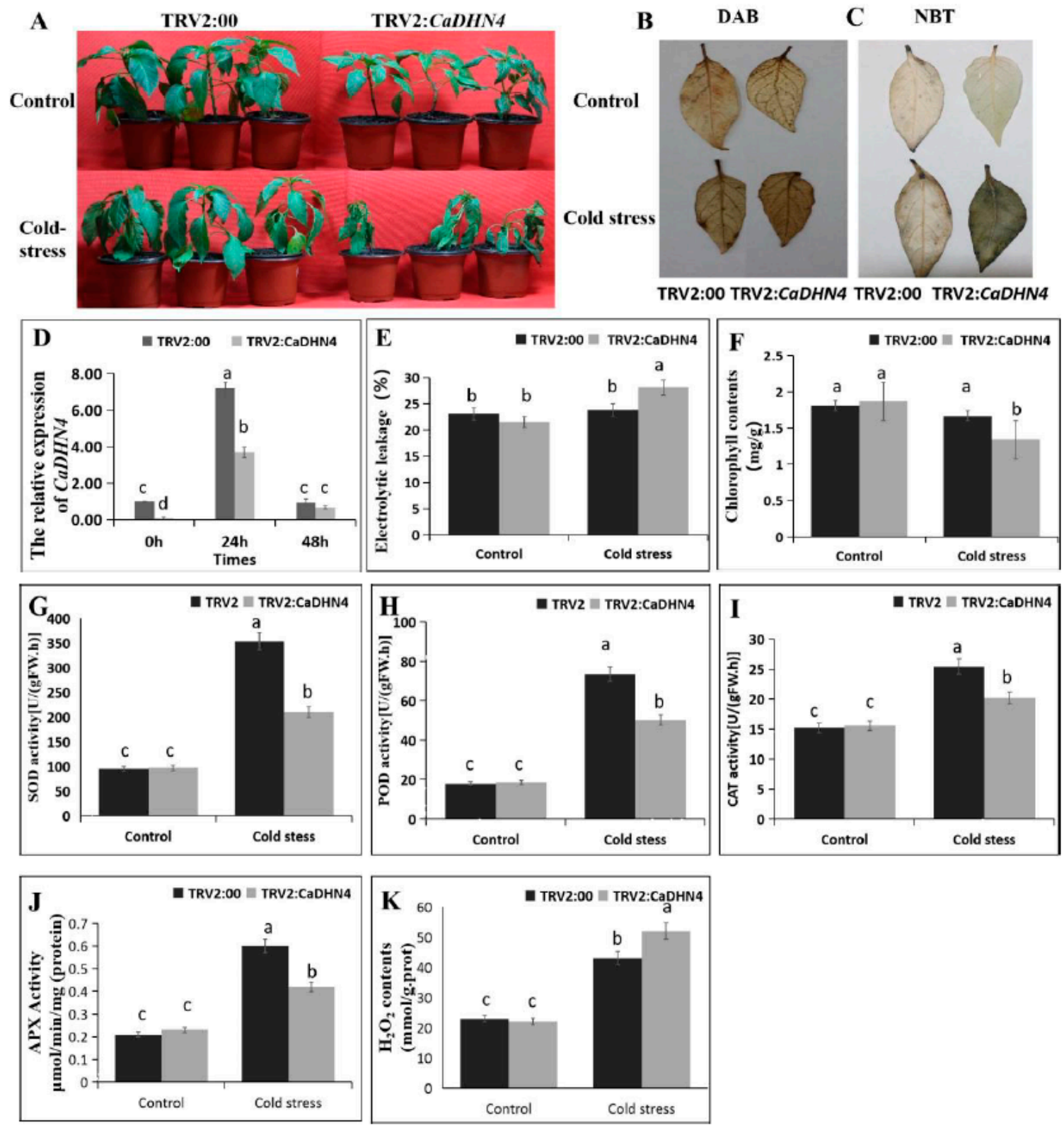

Figure 3. The phenotypes and analysis of CaDHN4 expression in gene-silenced pepper plants under cold stress. (A) The phenotype of silenced pepper plants under cold stress of 3 days. (B) 3,3'-Diaminobenzidine (DAB) staining. (C) Nitro-blue tetrazolium (NBT) staining. (D) The relative expression of CaDHN4 in silenced pepper plants under cold stress. (E) Electrolytic leakage rate. (F) Chlorophyll content. (G) Superoxide dismutase (SOD) activity. (H) Peroxidase (POD) activity. (I) Catalase (CAT) activity. (J) Ascorbate peroxidase (APX) activity. (K) $\mathrm{H}_{2} \mathrm{O}_{2}$ contents. Mean and S.D. values were obtained from three independent experiments, with 3 plants per experiments. The different small letters $(a-c)$ indicate significant differences at $p \leq 0.05$.

\subsection{Virus-Induced Gene Silencing (VIGS) of CaDHN4 Reduces Tolerance to Salt Stress}

To investigate the response of CaDHN4-silenced plants to salt tress, the pTRV2:00 and pTRV2:CaDHN4 plants were exposed to 200,300, and $400 \mathrm{mM} \mathrm{NaCl}$ for 3 days. We observed that the color of leaf discs gradually turned yellow with the increase of $\mathrm{NaCl}$ concentrations (Figure 4A); the leaf discs from pTRV2:CaDHN4 plants displayed more necrosis than the pTRV2:00 plants. With the increase of $\mathrm{NaCl}$ concentrations, the relative electrolyte leakage was higher in the pTRV2:CaDHN4 plants as compared to the pTRV2:00 plants (Figure 4B), and the total chlorophyll content of pTRV2:00 plants were higher than the pTRV2:CaDHN4 plants (Figure 4C). 
A
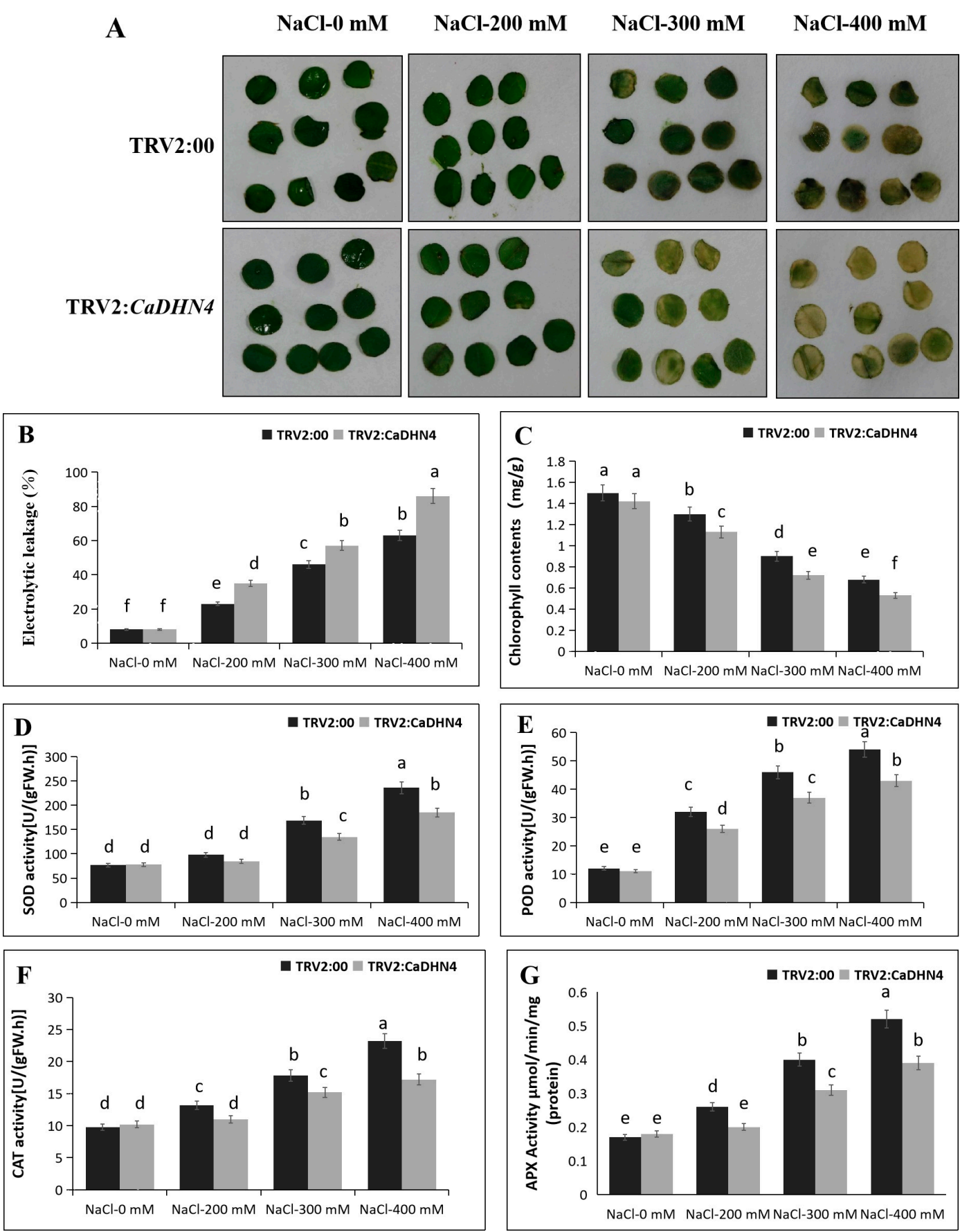

Figure 4. The measurement of physiological indices of CaDHN4-gene-silenced pepper plants under salt stress. (A) The phenotype of silenced pepper plants under salt stress in detached leaf discs. (B) Electrolytic leakage rate. (C) Chlorophyll content. (D) SOD activity. (E) POD activity. (F) CAT activity. (G) APX activity. Mean and S.D. values were obtained from three independent experiments, with 10 leaves per treatment, respectively. The different small letters (a-f) indicate significant differences at $p \leq 0.05$.

Then, we measured the activities of SOD, POD, CAT, and APX in pTRV2:CaDHN4 plants and the pTRV2:00 plants under normal and different $\mathrm{NaCl}$ concentrations. In normal condition, the activity of SOD, POD, CAT, and APX were not significantly different between control and silenced plants. While subjected to different $\mathrm{NaCl}$ concentrations, a remarkable increase in the activities of SOD, POD, CAT, and APX were observed in pTRV2:CaDHN4 and pTRV2:00 plants. However, this increase of enzymatic activities was higher in pTRV2:00 plants than the pTRV2:CaDHN4 plants (Figure 4D-G). 


\subsection{Overexpression of CaDHN4 in Arabidopsis Increases Cold Stress Tolerance}

To explore the response of CaDHN4-overexpressing plants to cold stress, we chose five lines of overexpressed transgenic lines of Arabidopsis. The relative expression of CaDHN4 of the lines of (27\# and 28\#) were significantly higher than three lines (3\#, 10\# and 16\#) (Figure 5B), so the transgenic Arabidopsis lines 27\# and 28\# were selected for the experiments. For cold stress, transgenic Arabidopsis and WT plants were exposed to $4{ }^{\circ} \mathrm{C}$ for 3 days. The WT plant leaves exhibited a significant higher water loss and morphological changes, whereas, the transgenic plants had green leaves (Figure 5A). To further illustrate the function of $\mathrm{CaDHN} 4$ in regulating tolerance to cold stress, we found that there were no significant difference in the malondialdehyde (MDA) content and relative electrolyte leakage between the WT and transgenic plants under normal growth conditions. However after cold treatment, the WT plants showed an increased level of MDA (3.8 folds) and relative electrolyte leakage (2-folds), as compared to the transgenic plants, where lower levels of MDA (1.6-2.7 folds) and relative electrolyte leakage (1.1 folds) were observed. These data showed that the electrolyte leakage and MDA contents were higher in WT, as compared to the transgenic plants (Figure 5C,D). In addition, to determine whether cold tolerance in $\mathrm{CaDHN} 4$ transgenic plants and interaction of cold-stress-related genes, including AtCOR47, AtDREB2A, AtRD29B, and AtERD7 assays were performed through qRT-PCR. The expressions of these genes were significantly higher in the CaDHN4 overexpressed plants than the WT plants (Figure 5E-H). Taken together, these results suggest that the overexpression of CaDHN4 enhanced cold tolerance in Arabidopsis.
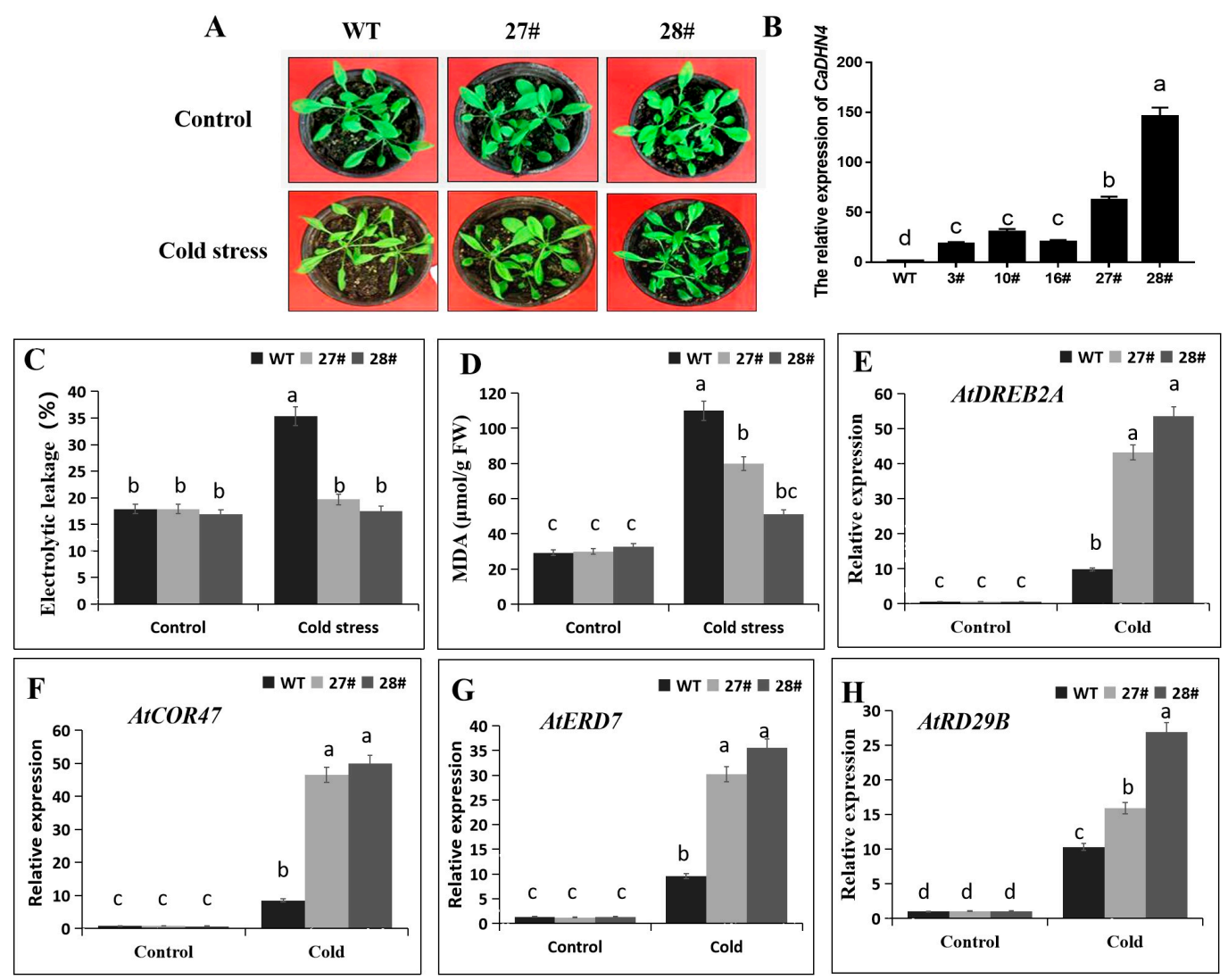

Figure 5. The measurement of physiological indices of CaDHN4-overexpressing transgenic and wild-type Arabidopsis under cold stress for 3 days. (A) Phenotypes of CaDHN4-overexpressing transgenic and wild-type Arabidopsis under cold stress. (B) Expression analysis of CaDHN4-overexpressing transgenic Arabidopsis plants by qRT-PCR. (C) Electrolytic leakage rate. (D) Malondialdehyde (MDA) content. (E-H) qRT-PCR analysis of cold-inducible genes in the CaDHN4 overexpression plants and WT plants in response to cold stress. Mean and S.D. values were obtained from three independent experiments, with 3 plants per treatment. The different small letters $(\mathrm{a}-\mathrm{d})$ indicate significant differences at $p \leq 0.05$. 


\subsection{Overexpression of CaDHN4 in Arabidopsis Increases Salt Stress Tolerance}

In order to determine the effect of $\mathrm{CaDHN} 4$ overexpression on salt-stress tolerance, the transgenic and WT Arabidopsis plants at different stages of development, namely seed germination, post-germination growth, and mature plants, were exposed to salt stress. We first examined the seed germination rates and post-germination primary root growth of transgenic and WT seeds on 1/2 MS medium or 1/2 MS medium containing $150 \mathrm{mM} \mathrm{NaCl}$. Transgenic plants had the higher seed germination rates than the WT (Figure 6B). To further illustrate the function of CaDHN4 in regulating tolerance to salt stress, on the third day, we found that the seed germination rates of transgenic plants were higher than WT plants with $150 \mathrm{mM}$ $\mathrm{NaCl}$ (Figure 6B). Next, we measured the fresh weight and found that under salt stress, the fresh weight of transgenic plants were higher ( 50-52\%) than the WT plants with $150 \mathrm{mM} \mathrm{NaCl}$ (Figure 6C).

A MS

$\mathrm{NaCl}-150 \mathrm{mM}$
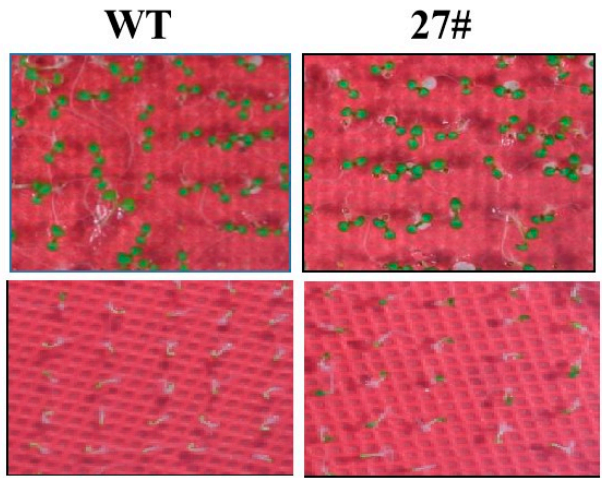

\section{8\#}

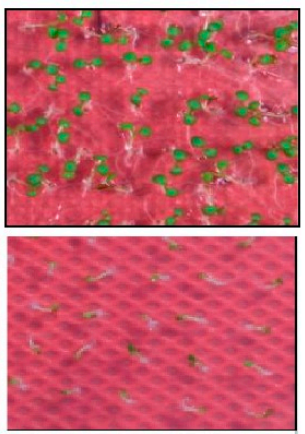

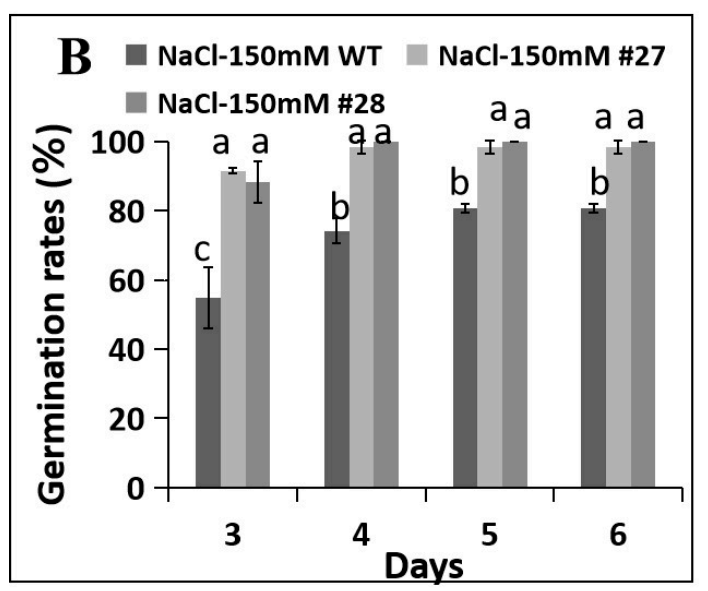

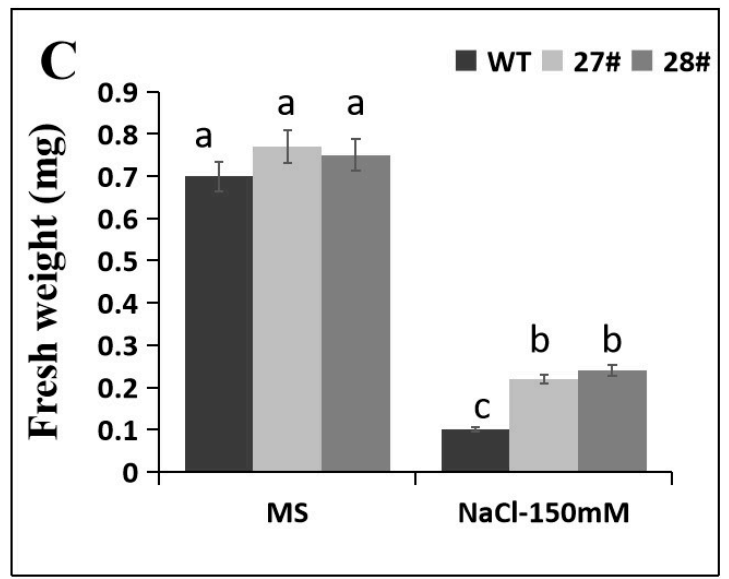

Figure 6. Analysis of salt resistance of CaDHN4-overexpressing transgenic and wild type Arabidopsis. (A) Phenotypes of CaDHN4-overexpressing plants under salt stress. (B) The seed germination rate of CaDHN4-overexpressing transgenic and wild type Arabidopsis under $150 \mathrm{mM} \mathrm{NaCl}$ treatment. (C) The fresh weight of CaDHN4-overexpressing transgenic and wild type Arabidopsis under $150 \mathrm{mM}$ $\mathrm{NaCl}$ treatment. Mean and S.D. values were obtained from three independent experiments, with 30 seeds per treatment. The different small letters $(\mathrm{a}-\mathrm{c})$ indicate significant differences at $p \leq 0.05$.

According to the observations of post-germination primary root growth under salt stress, no significant difference in the root lengths were noticed in the control conditions $(0 \mathrm{mM} \mathrm{NaCl})$ between the WT and transgenic plants. However, the root lengths of transgenic plants were higher $(\sim 40-60 \%)$ than the WT plants with $150 \mathrm{mM} \mathrm{NaCl}$ (Figure 7A,B). Next, we also measured the fresh weight and found that under salt stress, the fresh weight of transgenic plants were higher ( 50-70\%) than the WT plants with $150 \mathrm{mM}$ $\mathrm{NaCl}$ (Figure 7C). 
A

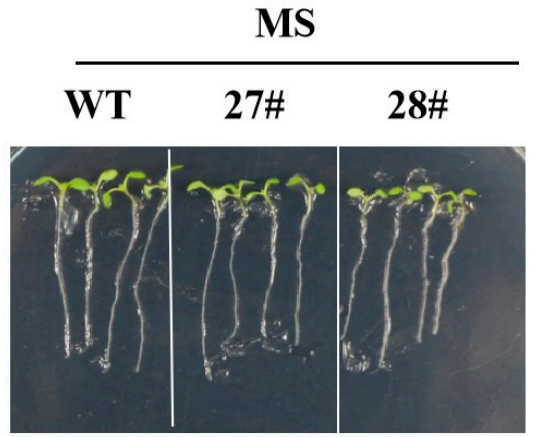

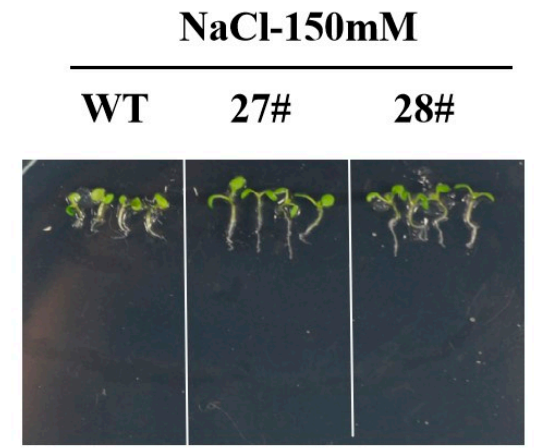
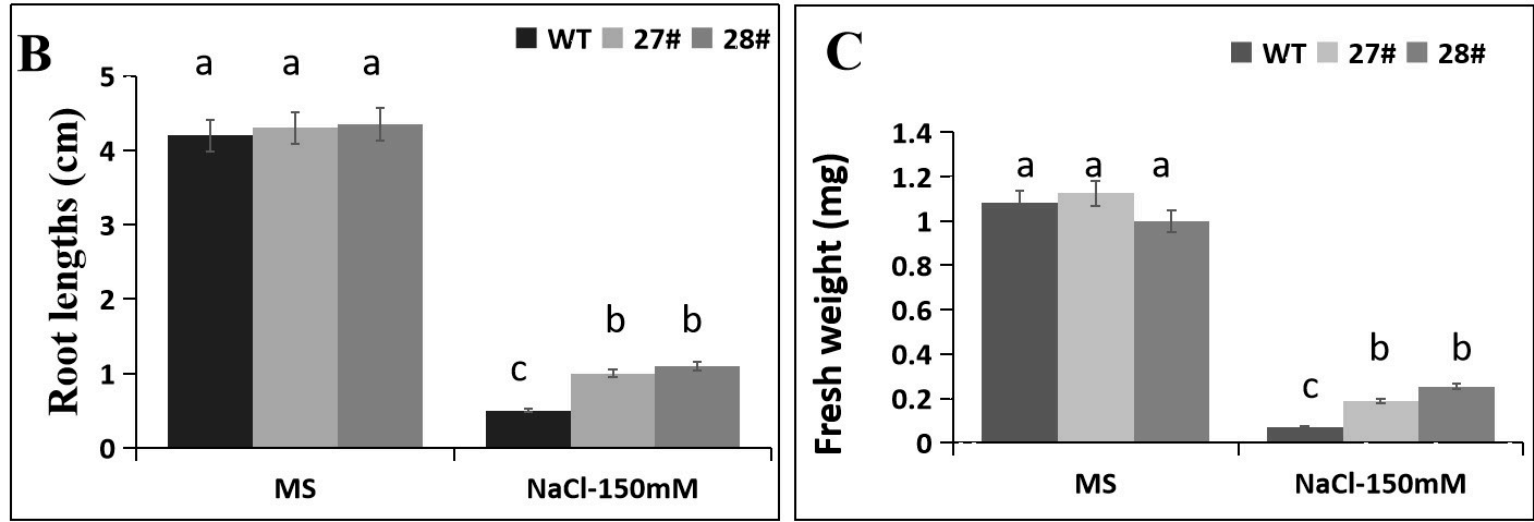

Figure 7. The root lengths of CaDHN4-overexpressing transgenic plants and wild type Arabidopsis under salt stress. (A) The phenotypes of different transgenic lines and WT under control (MS) or NaCl treatment. (B) The root lengths of CaDHN4-overexpressing transgenic plants and wild type Arabidopsis under $150 \mathrm{mM} \mathrm{NaCl}$ treatment. (C) The fresh weight of CaDHN4-overexpressing transgenic plants and wild type Arabidopsis under $150 \mathrm{mM} \mathrm{NaCl}$ treatment. Mean and S.D. values were obtained from three independent experiments, with 4 plants per treatment. The different small letters $(\mathrm{a}-\mathrm{c})$ indicate significant differences at $p \leq 0.05$.

We subsequently elucidated that overexpression of CaDHN4 in Arabidopsis improved the salt tolerance after $12 \mathrm{~h}$ exposure to $250 \mathrm{mM} \mathrm{NaCl}$. We found that after the salt stress, the WT plants exhibited severe wilted leaves phenotypes, while no evident wilting symptoms were observed for the CaDHN4 overexpressed Arabidopsis lines (Figure 8A). Under $250 \mathrm{mM} \mathrm{NaCl}$ treatments for $12 \mathrm{~h}$, the transgenic plants showed lower $(\sim 40-42 \%)$ MDA content and $(\sim 25-30 \%)$ lower relative electrolyte leakage as compared to WT plants (Figure 8B,D). In contrast, the leaf chlorophyll content of the transgenic plants were higher ( $13-14 \%$ ) than the WT plants (Figure $8 \mathrm{C}$ ). Through measurement of water loss rate, the transgenic plants showed lower rates of water loss than the WT plants, indicating that $\mathrm{CaDHN} 4$ transgenic plants have an increased water retention capacity (Figure 8E). In addition, qRT-PCR analysis was performed to determine the expression of salt-stress responsive genes, such as AtCOR47, AtDREB2A, AtRD29B, and AtERD7. Salt stress induced the expression of these genes in both the WT and CaDHN4 overexpressed Arabidopsis plants as compared to non-treated plants (Figure 8F-I). These marker genes expressed more in the leaves of CaDHN4 transgenic lines than the WT Arabidopsis plants. These data suggest that overexpression of $\mathrm{CaDHN4}$-enhanced tolerance to salt stress in CaDHN4 overexpressed Arabidopsis plants. 


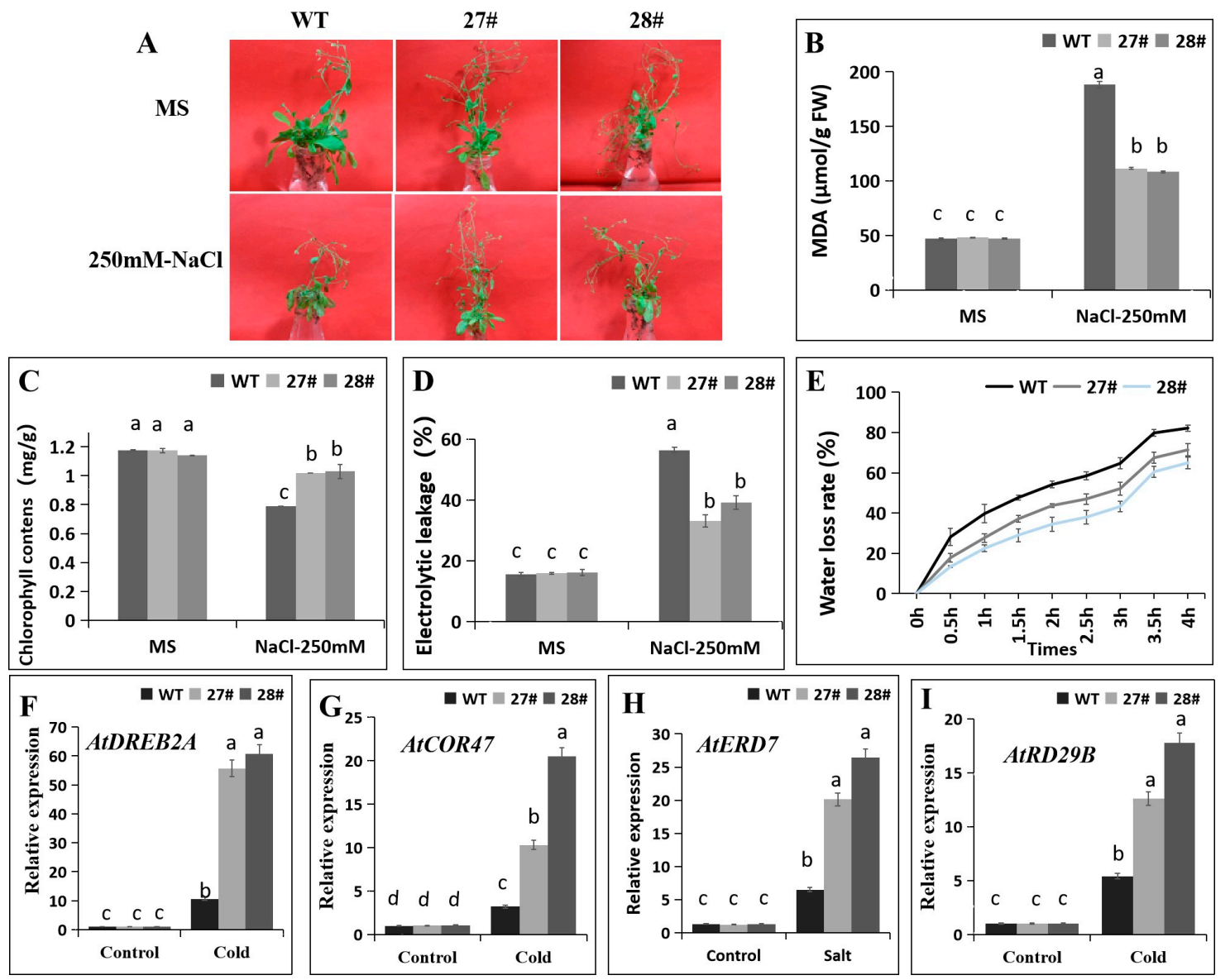

Figure 8. The measurement of physiological indices of CaDHN4-overexpressing transgenic plants and wild type Arabidopsis under salt stress at mature stage of growth. (A) The phenotypes of CaDHN4-overexpressing transgenic plants and wild type Arabidopsis under salt stress at mature stage. (B) MDA content. (C) Chlorophyll content. (D) Electrolytic leakage. (E) Water loss rate.(F-I) qRT-PCR analysis of salt-inducible genes in the CaDHN4 overexpression plants and WT plants in response to high salinity. Mean and S.D. values were obtained from three independent experiments, with 3 plants per treatment. The different small letters $(a-d)$ indicate significant differences at $p \leq 0.05$.

\subsection{Overexpression of CaDHN4 in Arabidopsis Decreases ABA Sensitivity}

In order to further confirm the function of $\mathrm{CaDHN} 4$, we overexpressed this gene in Arabidopsis. First, we randomly selected five transgenic lines (3\#, 10\#, 16\#, 27\#, and 28\#) from the T3 generations. Then, we screened these five lines on $0.5 \mu \mathrm{M}$ ABA, where 27\# and 28\# performed better than the other lines (Figure S2). In order to determine the role of CaDHN4 in the ABA response, we initially quantified seed germination rate in response to ABA. The seed germination assay revealed that no significant difference was observed in the seed germination rate between transgenic and WT Arabidopsis plants under normal conditions. However, we found that with exposure to 0.5 and $1 \mu \mathrm{M} \mathrm{ABA}$, the seeds from transgenic plants exhibited much higher germination rates than the WT (Figure 9E). With an increase in ABA concentration, the seed germination rate decreased; the seed germination rates of WT plants were lower than the transgenic plants (Figure 9E). Under 0.5 and $1 \mu \mathrm{M} \mathrm{ABA}$ treatments, the fresh weight of transgenic plants were higher than WT plants (Figure 9F). We further investigated the ABA sensitivity of the CaDHN4-overexpressing transgenic lines at the mature stage by floating the Arabidopsis leaves on 1/2 MS liquid medium supplemented with $50 \mu \mathrm{M}$ ABA. As shown in Figure 9B,D, WT plants almost became yellow after 3 days of treatment. The chlorophyll content also indicated that the WT plants lost approximately $90 \%$ of the chlorophyll, as compared to the leaves that were not treated with ABA. 
In contrast, in the transgenic Arabidopsis plants' leaves, yellow areas were lower than those in the WT plants, and lost only $40-60 \%$ of the chlorophyll contents, compared to the leaves without ABA treatments.

ABA treatment induced the excessive accumulation of ROS, which led to the oxidative stress. To know whether the ABA sensitivity is correlated with the ROS levels, the accumulation of hydrogen peroxide $\left(\mathrm{H}_{2} \mathrm{O}_{2}\right)$ and superoxide radical $\left(\mathrm{O}^{2-}\right)$ were measured through DAB and NBT staining, respectively (Figure 9). Under normal growth conditions, there was no significant difference in the ROS accumulation in the overexpressed lines and the WT plants, as shown by the comparable light color of DAB- and NBT-stained leaves. After the exposure to $50 \mu \mathrm{M}$ ABA, we found that the blue or brown colors were more intense in both the leaves of transgenic lines and WT plants, indicating more accumulation of ROS. However, the $\mathrm{CaDHN}$-overexpression lines accumulated less $\mathrm{H}_{2} \mathrm{O}_{2}$ and $\mathrm{O}^{2-}$ than the WT plants (Figure 9C).

Next, we measured the activities of three main antioxidant enzymes, i.e., SOD, POD, and CAT in $\mathrm{CaDHN} 4$ overexpressed lines and the WT plants under normal and/or $50 \mu \mathrm{M}$ ABA conditions (Figure 9G-J). No significant differences were observed for the activities of these three antioxidant enzymes under normal conditions. However, when subjected to $50 \mu \mathrm{M}$ ABA, a remarkable increase in the activities of SOD, POD, CAT, and APX were observed in both the transgenic Arabidopsis lines and WT plants. However, the higher enzymatic activities were more pronounced in the overexpression Arabidopsis lines as compared to the WT plants. In addition, we also measured the $\mathrm{H}_{2} \mathrm{O}_{2}$ contents under ABA treatment through NBT and DAB staining. No significant difference in $\mathrm{H}_{2} \mathrm{O}_{2}$ contents under normal conditions were noticed; however, under $50 \mu \mathrm{M} \mathrm{ABA}$, the $\mathrm{H}_{2} \mathrm{O}_{2}$ contents in overexpressed Arabidopsis lines were lowered than the WT plants (Figure 9K).

Then, qRT-PCR analysis was performed to examine how the CaDHN4 overexpression affects the ABA signaling and biosynthesis. Treatment with $50 \mu \mathrm{M}$ ABA highly induced the ABA responsive genes such as AtAFB3 and AtNCED3 in 3 week-old CaDHN4 overexpressed Arabidopsis and WT plants. The expression levels of these genes were lower in the CaDHN4 overexpressed Arabidopsis than the WT plants (Figure $9 \mathrm{~L}, \mathrm{M}$ ). This suggests that the CaDHN4 gene plays an important role in ABA-induced gene expression.

Next, we measured the ABA-induced stomatal conductance. Leaves from 3-week-old Arabidopsis plants were incubated in stomatal opening solution and treated with different concentrations $(0,5$, 10 , and $20 \mu \mathrm{M}$ ) of $\mathrm{ABA}$ for $2 \mathrm{~h}$, and then we measured the stomatal aperture. We found that no obvious difference in stomatal aperture between WT and transgenic Arabidopsis lines under $0 \mu \mathrm{M}$ ABA (Figure 10A). However, after treatment with ABA for $2 \mathrm{~h}$, the stomatal apertures of the WT plants were smaller ( 30-70\%) than the CaDHN4-overexpressed transgenic Arabidopsis plants (Figure 10B). These results showed that $\mathrm{CaDHN} 4$ decreased sensitivity to $\mathrm{ABA}$. 
A

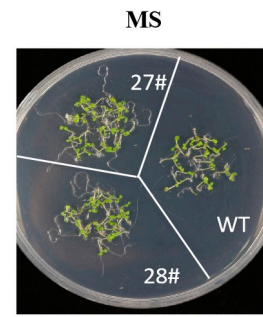

C
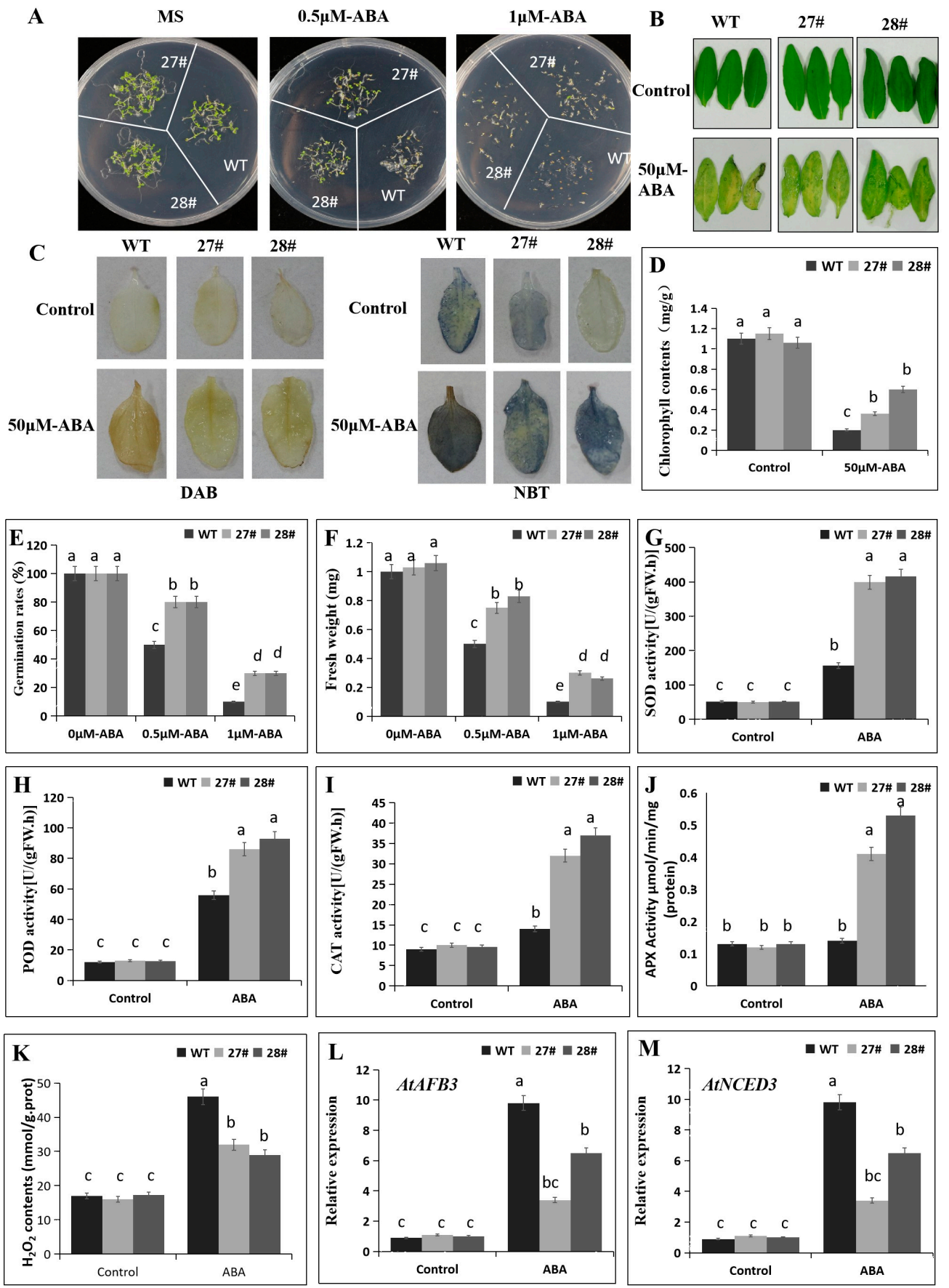

Figure 9. The effect of ABA on seed germination and leaf greening in CaDHN4-overexpressing Arabidopsis plants. (A) The phenotypes of CaDHN4-overexpressing plants under ABA treatment. (B) Photographs of detached leaves from 4-week-old transgenic Arabidopsis and WT plants floated on 1/2 MS medium or 1/2 MS medium containing $50 \mu \mathrm{M}$ ABA for 3 days. (C) DAB and NBT staining. (D) Chlorophyll content of CaDHN4-overexpressing transgenic and wild type Arabidopsis plants under 1/2 MS medium or 1/2 MS medium containing $50 \mu \mathrm{M}$ ABA. (E) The seed germination rate of $\mathrm{CaDHN4}$-overexpressing transgenic and wild type Arabidopsis plants under different concentrations of ABA. (F) The fresh weight of CaDHN4-overexpressing transgenic and wild type Arabidopsis plants under different concentrations of ABA. (G) SOD activity. (H) POD activity. (I) CAT activity. ( J) APX activity. (K) $\mathrm{H}_{2} \mathrm{O}_{2}$ contents. (L,M) qRT-PCR analysis of ABA responsive genes AtAFB3 and AtNCED3 in the CaDHN4 overexpression plants and WT plants after ABA treatment. Mean and S.D. values were obtained from three independent experiments, with 30 seeds and 3 leaves per treatment. The different small letters $(\mathrm{a}-\mathrm{c})$ indicate significant differences at $p \leq 0.05$. 
A

है
WT

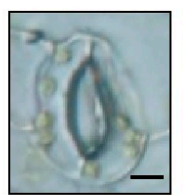

5.0
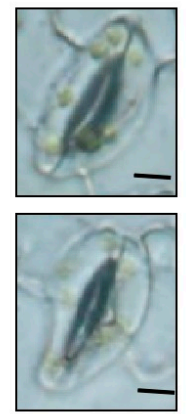

20.0

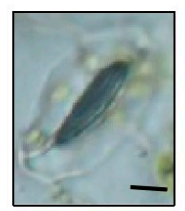

27\#
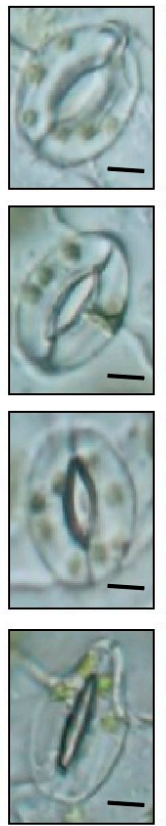

28\#
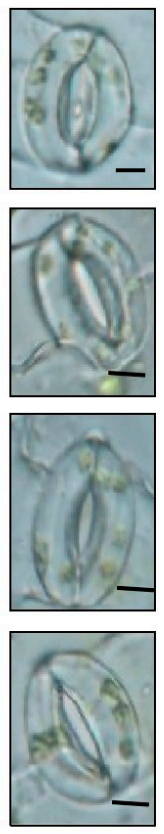

B

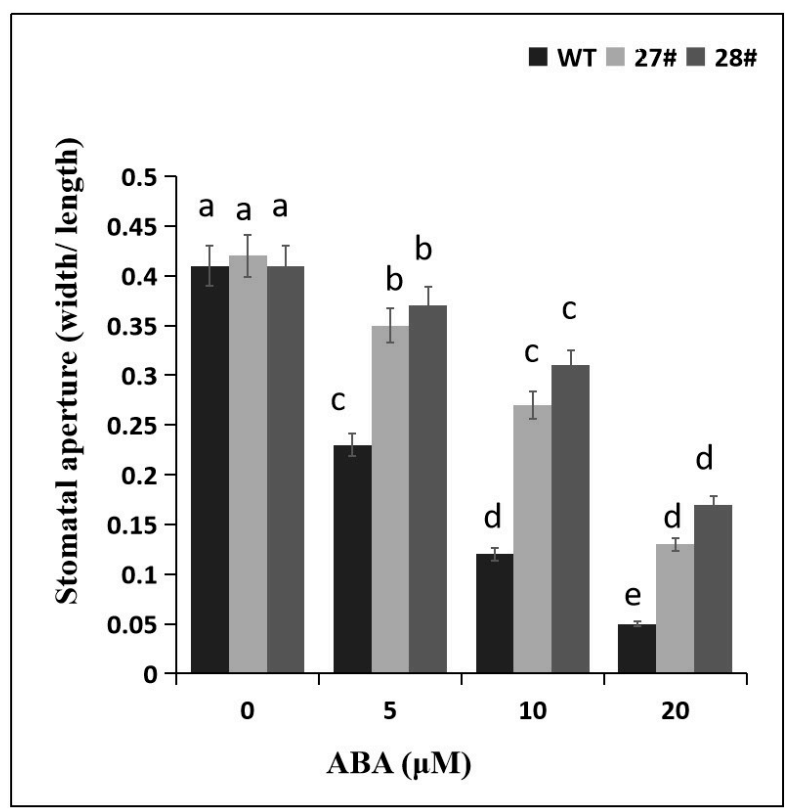

Bar $=20 u m$

Figure 10. Alterations in stomatal aperture of CaDHN4 transgenic Arabidopsis in response to ABA treatments. (A) Stomatal aperture of CaDHN4 transgenic and WT plants in response to ABA. (B) Stomatal aperture values. Mean and SD values were obtained from three independent experiments. The different small letters (a-e) indicate significant differences at $p \leq 0.05$.

\section{Discussion}

DHNs have a protective effect on the low temperature and can maintain the stability of cell membranes by preventing the lipid peroxidation and also scavenging of the reactive oxygen species [32]. To understand the regulatory mechanism of CaDHN4 under different stresses, virus-induced gene silencing (VIGS) of CaDHN4 was performed in pepper. Under cold stress, there was more serious wilting in the pTRV2:CaDHN4 silenced pepper plants than the pTRV2:00 pepper plants. Electrolyte leakage and chlorophyll contents in leaves are often used as indicators of plant membrane injury under abiotic stresses [33]. MDA, the product of lipid peroxidation caused by reactive oxygen species (ROS), are used to evaluate ROS-mediated injuries in the plants [34]. The electrolyte leakage and chlorophyll contents also showed that in the PTRV2:CaDHN4 plants, membrane damage and leaf senescence were more serious under low temperature stress. Thus, MDA content and electrolyte leakage were measured to assess the role of CaDHN4 in the overexpressed Arabidopsis plants, which reduced the membrane injury under cold and salt stresses. The level of electrolyte leakage in the WT were significantly higher than the transgenic Arabidopsis plants under cold and salt stresses (Figures 5 and 8), and the MDA content in the WT plants were significantly higher than the transgenic plants under cold and salt stresses. Silencing of CaDHN4 in pepper, the electrolyte leakage displayed an opposite pattern. These results were consistent with those described by Chen et al. [35].

The DAB and NBT staining results showed more intense staining in the pTRV2:CaDHN4 pepper plants, as compared to the pTRV2:00 pepper plants. These data indicated that silencing of CaDHN4 resulted in higher accumulation of hydrogen peroxide and superoxide anions under low temperature stress. To test the regulatory mechanisms of CaDHN4 and further understand the function of $\mathrm{CaDHN} 4$, we generated CaDHN4-overexpressed Arabidopsis plants and determined their responsiveness to cold and salt stresses. Under normal conditions, there were no obvious differences in the plant growth, morphology, and physiological indices between the transgenic Arabidopsis and WT plants. 
After cold stress treatment for 3 days, the transgenic Arabidopsis plants grew better than the WT plants. These results indicated that the degree of cell membrane damage and lipid peroxidation of transgenic Arabidopsis plants were lower than the WT plants, under low temperature stress. Our results are consistent with the overexpression of maize $\mathrm{ZmDHN} 2 b$ in tobacco, which resulted in the higher electrolyte leakage and MDA content in transgenic plants, which were lower than the WT plants under $4{ }^{\circ} \mathrm{C}$ treatment [36]. In wheat, overexpression of the acidic dehydrin WCOR410 improved the freezing tolerance in the strawberry leaves [37].

When plants are subjected to abiotic stresses, they accumulate more ROS that may cause oxidative damage to the biomolecules in the plants [38], so the ROS scavenging is closely related to the plants' tolerance to abiotic stresses. To know the ROS scavenging mechanism, we tested the accumulation of $\mathrm{H}_{2} \mathrm{O}_{2}$. The results showed that under cold stress, the pTRV2:CaDHN4 pepper plants had higher $\mathrm{H}_{2} \mathrm{O}_{2}$ contents as compared to the pTRV2:00 pepper plants. While, under ABA treatment the overexpressed Arabidopsis lines displayed an opposite pattern. In addition, SOD, POD, CAT, and APX as the antioxidant enzymes involved in ROS scavenging, can scavenge $\mathrm{H}_{2} \mathrm{O}_{2}$. We measured SOD, POD, CAT, and APX activities under cold, salt, and ABA treatment. The results showed that under cold stress, the pTRV2:00 pepper plants had higher SOD, POD, CAT, and APX activities than pTRV2:CaDHN4 silenced pepper plants. In contrast, under ABA treatment, the overexpressed Arabidopsis lines had lowered enzymatic activities than the WT plants. These results indicated that silencing of CaDHN4 reduced the activities of SOD, POD, CAT, and APX activities by inhibiting the expression of antioxidant related genes in vivo, leading to the less accumulation of ROS in plants, resulting in reduced tolerance of plants to cold stress; whereas, the ABA treatment displayed an opposite pattern in Arabidopsis.

Under salt stress, the results showed that the germination rate, fresh weight, chlorophyll contents, and root lengths of transgenic plants were higher than the WT plants; whereas, the MDA content and electrolyte leakage of the transgenic plants were lower. These results are in agreement with studies in other plants such as wheat, where overexpression of TaDHN1 and TaDHN3 resulted in the better growth and longer roots than the control plants under salt stress [29]. CaDHN4-overexpressed Arabidopsis plants displayed tolerance and a better growth under cold and salt stresses. These results demonstrated that $\mathrm{CaDHN} 4$ is a positive regulator of cold and salt stresses tolerance in Arabidopsis. The mechanisms responsible for improved cold and salt tolerance in Arabidopsis might be due to several beneficial changes at the morphological and physiological level. Additionally, overexpression of CaDHN4 in Arabidopsis resulted in higher seed germination rate than WT plants with exogenous ABA treatment. These data suggested that overexpression of CaDHN4 in Arabidopsis decreased the sensitivity to exogenous ABA, which endorses the previous findings of Fujita et al. [39].

Taken together, these data showed that CaDHN4-overexpressed Arabidopsis plants exhibited a decreased lipid peroxidation and membrane injury under salt- or cold-stress conditions than the WT plants. Plants possess a very efficient enzymatic antioxidant defense system to protect cells from oxidative damage by scavenging ROS, such as SOD, POD, CAT, and APX. According to this study, showed that $\mathrm{CaDHN} 4$ gene maintained the stability of cell membranes, prevented lipid peroxidation and lowered the accumulation of reactive oxygen species, thereby playing an important role in low temperature stress tolerance.

The cis-acting elements in promoters play a crucial role in the regulation of gene transcription [40]. Many DHN genes and their promoters have been identified, and their regulatory pathways have been well discovered [27,41,42]. Analysis of GUS activity of the promoter of CaDHN4 in transgenic Arabidopsis under different stress conditions found that low temperature, $\mathrm{NaCl}$, mannitol, drought, MeJA, SA, and ABA treatments, resulted in intense staining in the transgenic plants, as compared to the control plants. These results are consistent with studies on barley OsDHN1 gene promoter [43], banana MusaDHN1 promoter [28], and three wheat DHN promoters (TaDHN1, TaDHN2, and TaDHN3) [29]. These results showed that $C a D H N 4$ are involved in pathways related to cold and salt stresses.

Overexpression of $\mathrm{CaDHN} 4$ induced the expression of the other stress-related genes including AtERD7, AtCOR47, AtRD29B, and AtDREB2A (Figure 9). These genes are involved in the mediation 
of osmosis and/or oxidative damage. For example, $A t R D 29 B$ encodes a hydrophilic protein that promotes water retention and maintains membrane integrity [44]. AtDREB2A is a member of the AP2/ERF family, and plays an important role in cold stress [45]. Increased expression of these genes (AtERD7, AtCOR47, AtRD29B, and AtDREB2A) in CaDHN4 can help plants to cope with the adverse environmental conditions.

We selected lines $27 \#$ and $28 \#$ for further investigations based on the screening results on $0.5 \mu \mathrm{M}$ ABA and expression analysis (Figure S2) of randomly selected 5 transgene linesfrom T3 generations. Our selection of two lines for further studies is consistent with Huang et al. [46], Guan et al. [47], and Shi et al. [48]. ABA act as an important phytohormone in the plant responses to abiotic stresses [49], and several studies have shown that ABA sensitivity correlate with stress tolerance in plants [50-52]. Our results demonstrated that overexpression of CaDHN4 in Arabidopsis decreased the ABA sensitivity, perhaps through the ABA-mediated signaling pathway. This was supported by the $\mathrm{CaDHN} 4$ promoter: GUS genes in transgenic plants were expressed under ABA hormone treatments (Figure 2B). CaDHN4 overexpression also resulted in sensitivity to ABA. The germination rate of $C a D H N 4$ overexpressing lines was higher than the WT plants after exposure to exogenous ABA (Figure 9). Expression of the ABA-responsive genes AtNCED3 and AtAFB3 were significantly decreased in the CaDHN4-overexpressed Arabidopsis lines as compared to the WT plants (Figure 9). These data suggest that $\mathrm{CaDHN} 4$ might participate in the regulation of the expression of ABA biosynthesis-related genes. The results showed that $\mathrm{CaDHN} 4$ expression negatively regulates ABA signaling by decreasing the ABA sensitivity of plant growth and restraining the induced expression of ABA-responsive genes. Based on the results, we found that although the threshold level of transcripts was different, both independent transgenic lines displayed similarly, such as the phenotypic change, the root length, the germinate rate and so on. The two overexpression lines showed different responses in some of the assays, which may be due to the difference in the relative expressions of the two overexpressed lines. The higher the expression, the stronger was the resistance; on the contrary, the lower the expression, the lower was the resistance. It can be seen in the Figure 5A, Figure 6A, and Figure 8A. The results are consistent with Zhai et al. [53], i.e., the higher expression of OE\#4 has stronger drought and salt-stress tolerance in Arabidopsis than the lower expression of OE\#1, and Cao et al. [23], i.e., the higher expression has stronger abiotic stress responses in Aarabidopsis. The level of expression may be relative to the position effect resulting gene silencing [54], DNA methylation [55], and so on.

On the other hand, over expression of CaDHN4 increased the salt tolerance in overexpression lines. We speculate that after overexpression of CaDHN4 in Arabidopsis, it might have induced the other signaling pathways, as the ABA regulatory mechanism is relatively complex. The enhanced salt tolerance in CaDHN4-overexpressing Arabidopsis plants could be due to direct/indirect regulation of stress-responsive genes through an ABA-mediated pathway. In summary, CaDHN4 has a role in the ABA-dependent signaling transduction pathways that induce cold and salt stress tolerance in Arabidopsis

Taken together, this study provides substantial evidence that this gene might be involved in cold and salt stress tolerance, and decrease ABA sensitivity in Arabidopsis.

\section{Materials and Methods}

\subsection{Plant Materials and Growth Conditions}

The Arabidopsis Wild type Col-0 and pepper cold resistant cultivar 'P70' provided by College of Horticulture, Northwest A\&F University, Yangling, China $\left(34^{\circ} 20^{\prime} \mathrm{N} 108^{\circ} 24^{\prime} \mathrm{E}\right)$; were used in this study. Pepper plants were grown at $22{ }^{\circ} \mathrm{C}$ with $75 \%$ relative humidity and no supplemental light. The transgenic Arabidopsis plants were grown at $22{ }^{\circ} \mathrm{C}$ with $75 \%$ relative humidity under long-day (16 h of light at $125 \mu \mathrm{mol} \cdot \mathrm{m}^{-2} \cdot \mathrm{s}^{-1}$ and $8 \mathrm{~h}$ of dark) conditions. 


\subsection{RNA Isolation and $q R T-q P C R$ Analysis}

Total RNA was extracted by using the Tiangen RNA extraction kit (TianGen, Xi'an, China), according to the manufacturer's protocol, and the synthesis of cDNA and qRT-PCR were executed as described by Chen et al [56]. Relative expression levels were analyzed according to the $2^{-\Delta \Delta C T}$ method [57]. ACTIN2 was used as an internal control. The primer sequences of qRT-PCR are listed in Table S2.

\subsection{Isolation and Sequence Analysis of CaDHN4}

Full-length of CaDHN4 (519bp) was amplified from the template cDNA of pepper cultivar P70, and CaDHN4 (CA02g22060) was obtained by pepper genome database (http://peppergenome. snu.ac.kr/). The forward and reverse primers for full-length of CaDHN4 cDNA sequences were 5'ATGTCGCACTACGAGAACCA3' and 5' CTACTAGTGGTGGCCAGTGCC $3^{\prime}$, respectively. PCR products were cloned into the pMD19-T vector (TaKaRa, Dalian, China) and sequenced (Shanghai GeneCore Biotechnologies Co. Shanghai, China).

\subsection{Promoter Activity Assay Analysis}

The gene-specific primers F1(5'-GACTAGTAGATTTTAATTTGCATGTATGAGC-3' SpeI site underline) and R1(5'-CCGCTCGAGCTTTCTGAACTAAGAACTGACCG-3' XhoI site underline) were used to amplify the CaDHN4 transcription start site, and then linked with cloning vector pMD-19T (Takara), and after re-sequencing, inserted into vector pBI121, and the recombinant plasmid pBI121-CaDHN4-GUS was obtained. For analyzing the promoter activity of CaDHN4 under abiotic stresses, 3-week-old plants were selected with $4^{\circ} \mathrm{C}, 100 \mathrm{mM} \mathrm{NaCl}, 250 \mathrm{mM}$ mannitol, $100 \mu \mathrm{M}$ MeJA, $100 \mu \mathrm{M} \mathrm{SA}$, and $50 \mathrm{mg} / \mathrm{L} \mathrm{ABA}$ for $24 \mathrm{~h}$ for GUS staining. The samples were stained in GUS staining buffer at $37^{\circ} \mathrm{C}$ for $24 \mathrm{~h}$ and de-colored with $75 \%$ alcohol, and then viewed and photographed under a microscope.

\subsection{Subcellular Localization Assays}

The gene-specific primers F2 (5'-GCTCTAGAATGTCGCACTACGAGAACCA-3' XbaI site underline) and R2 (5'-CGGGATCCCTAGTGGTGGCCAGTGCC-3' KpnI site underline) were used to amplify the $\mathrm{CaDHN} 4$ coding region without stop codon (TGA) and then linked with cloning vector pMD-19T (Takara), and after re-sequencing, inserted into vector pBI221, and the recombinant plasmid pBI221-CaDHN4-GFP was obtained. The empty vector pBI221-GFP was used as a control. The confocal laser-scanning microscope was used for GFP fluorescence detection (Nikon, Tokyo, Japan).

\subsection{Virus-Induced Gene Silencing (VIGS) of CaDHN4}

To silence CaDHN4, virus-induced gene silencing (VIGS) constructs, pTRV1 and pTRV2 vectors (tobacco rattle virus), were used. They were amplified by the gene-specific primers F3 (5'-GCTCTAGAATTGTGTAGTACGGTCAGTTCTT-3' XbaI site underline) and R3 (5'-CGGGATCC CAGTTGAATGGGCTTGGTC-3' BamHI site underline), then linked with cloning vector pMD-19T (Takara), and after re-sequencing, inserted into vector pTRV2, and the recombinant plasmid pTRV2:CaDHN4 was obtained. Agrobacterium tumefaciens GV3101 containing pTRV1 or pTRV2:CaNDHN4 were injected into pepper plants and plants were grown under conditions as described by Guo et al [58].

\subsection{Generation of CaDHN4 Transgenic Arabidopsis Plants}

Gene-specific primers F4 (5'-GCTCTAGAATGTCGCACTACGAGAACCA-3' $\underline{X b a I}$ site underline) and R4 (5'-CGGGATCCCTACTAGTGGTGGCCAGTGCC-3' KpnI site underline) were designed to amplify the coding region of CaDHN4, then linked with cloning vector pMD-19T (Takara), and after re-sequencing, inserted into vector pVBG2307, and the recombinant plasmid pVBG2307-CaDHN4 was obtained. The pVBG2307-CaDHN4 was introduced into the Agrobacterium tumefaciens strain GV3101 
by electroporation. Transgenic Arabidopsis plants were obtained using the floral dipping methods. Transgenic plants were screened by 1/2MS medium supplemented with $50 \mathrm{mg} / \mathrm{L}$ kanamycin, and the $\mathrm{T}_{3}$ generation was harvested for further use.

\subsection{Salt and Cold Stress Tolerance Assays}

For salt treatment, the transgenic Arabidopsis plants of seed germination rate and root length, and seeds from $\mathrm{T}_{3}$ transgenic Arabidopsis plants and WT were surface-sterilized and sown on 1/2 MS medium or $1 / 2 \mathrm{MS}$ medium containing $150 \mathrm{mM} \mathrm{NaCl}$ for 1 week: plants were grown with a $16 \mathrm{~h}$ light $/ 8 \mathrm{~h}$ dark cycle at $22{ }^{\circ} \mathrm{C}, 30$ seeds from each line; at maturity, the 4-week-old WT and transgenic Arabidopsis plants were immersed in $250 \mathrm{mM} \mathrm{NaCl}$ for $12 \mathrm{~h}$. For silencing of CaDHN4 through VIGS, the pepper leaf discs of TRV2:00 and TRV2:CaDHN4 were immersed in different concentrations of $\mathrm{NaCl}$ solution $(0,200,300$, and $400 \mathrm{mM})$ with continuous illumination for 3 days, with each treatment containing 10 pepper seedlings [53]. For cold treatment, the overexpressing Arabidopsis plants, WT plants, TRV2:00, and TRV2:CaDHN4 plants were treated at $4{ }^{\circ} \mathrm{C}$ for 3 days.

\subsection{Determination of Chlorophyll, MDA, and Relative Electrolyte Contents and Antioxidant Enzyme Activities}

The malondialdehyde (MDA) contents were determined following Dhindsa et al [59]; electrolyte leakage contents were measured as described by Dionisio-Sese and Tobita [60]; chlorophyll contents were measured as described by Arkus et al [61]. The activity of peroxidase (POD), superoxide dismutase (SOD), and catalase (CAT) was detected according to Liang et al [62]; the APX activity was assayed as previously described [63]; the $\mathrm{H}_{2} \mathrm{O}_{2}$ content was determined by the method of titanium oxidation with hydrogen peroxide-titanium complex formation [64].

\subsection{NBT and DAB Staining}

To determine the accumulation of hydrogen peroxide $\left(\mathrm{H}_{2} \mathrm{O}_{2}\right)$ and superoxide $\left(\mathrm{O}^{2-}\right)$ under cold stress, 3,3' -diaminobenzidine (DAB) and nitro-blue tetrazolium (NBT) staining were performed [65,66]. Each treatment, leaves were collected and cultivated in DAB (1 mg mL ${ }^{-1}$, pH 3.8) or NBT solution $\left(0.1 \mathrm{mg} \mathrm{mL}^{-1}\right)$ overnight at $25{ }^{\circ} \mathrm{C}$. After staining, the chlorophyll was removed by soaking in $75 \%$ ethanol and boiled for about $15 \mathrm{~min}$. After sufficient bleaching, the images were captured.

\subsection{ABA Tolerance Assays}

Germination assays were performed on seeds from $\mathrm{T}_{3}$ transgenic lines and WT plants. Seeds were surface-sterilized and sown on $1 / 2 \mathrm{MS}$ medium with or without $0.5 \mu \mathrm{M}$ ABA and $1 \mu \mathrm{M}$ ABA supplementation, and the germination rates of at least 30 seeds from each line were recorded daily. Additionally, the chlorophyll contents of detached leaves were measured. For the ABA sensitivity assay, leaves from 4-week-old soil-grown plants from each line were floated abaxial side up in 1/2 MS liquid medium with or without $50 \mu \mathrm{M}$ ABA for 3 days at room temperature with a $16 \mathrm{~h}$ light/8 h dark cycle followed by chlorophyll content measurement [67].

\subsection{Measurement of Stomatal Aperture in Response to ABA Treatment}

For the ABA-induced stomatal aperture measurement, leaves from 3-week-old T3 transgenic and WT plants were incubated in stomatal opening solution (10 mM KCl, $10 \mathrm{mM} \mathrm{MES-Tris,} \mathrm{pH} \mathrm{6.15,}$ and $\left.50 \mu \mathrm{M} \mathrm{CaCl}_{2}\right)$. After incubation for $2 \mathrm{~h}$, leaves were treated for $2 \mathrm{~h}$ with different concentrations of $\operatorname{ABA}(0,5,10$, and $20 \mu \mathrm{M})$. Then taking pictures with a light microscope and the stomatal apertures were measured using Image J software. Stomatal aperture values are presented as means from at least 50 stomata. 


\subsection{Statistical Analysis}

All data were analyzed by one-way ANOVA (Analysis of variance) using the SPSS (Chicago, IL, USA), and the means were compared using Tukey's HSD (Honest significant difference, Chicago, IL, USA) multiple range test, taking $p \leq 0.05$ as a significant difference.

\section{Conclusions}

In this study, we isolated a dehydrin gene from Capsicum annuum leaves, designated as CaDHN4. To verify the functional role of CaDHN4 in abiotic stresses tolerance, CaDHN4 was overexpressed in Arabidopsis and silenced in pepper plants through VIGS. Silencing of CaDHN4 decreased pepper cold and salt tolerance, but CaDHN4-overexpressing in Arabidopsis enhanced cold-and salt-stress tolerance. Moreover, overexpression of CaDHN4 in Arabidopsis decreased the ABA sensitivity. The level of expression may be relative to the position effect, resulting in gene silencing and DNA methylation. In the future experiments, we will authenticate it. Taken together, the results of our study showed that CaDHN4 might act as a positive regulator of cold- and salt-stress tolerance. This study will provide further insight for functional analysis of DHN genes in solanaceous and other crop species for adaptability to various stress conditions.

Supplementary Materials: Supplementary materials can be found at http://www.mdpi.com/1422-0067/21/1/26/s1.

Author Contributions: H.-f.Z., J.-h.M., and R.-g.C. conceived and designed the experiments; H.-f.Z., X.-k.W., and S.-y.L. performed the experiments; S.u.H. and Y.-c.M. analyzed the data; Y.-m.Z. contributed reagents/materials/ analysis tools; H.-f.Z. wrote the paper. All authors have read and agreed to the published version of the manuscript.

Funding: This research was funded by the National Natural Science Foundation of China (\#31672146, \#31201615) and the Natural Science Foundation of Shaanxi Province (2018JM3023).

Acknowledgments: We would like to thank the native English speaking scientists of Elixigen Company (Huntington Beach, California) for editing our manuscript.

Conflicts of Interest: The authors declare no conflict of interest.

\section{References}

1. Mittler, R. Abiotic stress, the field environment and stress combination. Trends Plant Sci. 2006, 11, 15-19. [CrossRef] [PubMed]

2. Jing, H.; Li, C.; Ma, F.; Ma, J.H.; Khan, A.; Wang, X. Genome-Wide Identification, Expression Diversication of Dehydrin Gene Family and Characterization of CaDHN3 in Pepper (Capsicum annuum L.). PLoS ONE 2016, 11, e0161073. [CrossRef] [PubMed]

3. Black, M.; Corbineau, F.; Gee, H.; Côme, D. Water content, raffinose, and dehydrins in the induction of desiccation tolerance in immature wheat embryos. Plant Physiol. 1999, 120, 463-471. [CrossRef] [PubMed]

4. Cellier, F.; Conéjéro, G.; Breitler, J.C.; Casse, F. Molecular and physiological responses to water deficit in drought-tolerant and drought-sensitive plants of sunflower accumulation of dehydrin transcripts correlates with tolerance. Plant Physiol. 1998, 116, 319-328. [CrossRef] [PubMed]

5. Guy, C.; Haskell, D.; Neven, L.; Klein, P.; Smelser, C. Hydration-state-responsive proteins link cold and drought stress in spinach. Planta 1992, 188, 265-270. [CrossRef] [PubMed]

6. Ingram, J.; Bartels, D. The molecular basis of dehydration tolerance in plants. Annu. Rev. Plant Physiol. Plant Mol. Biol. 1996, 47, 377-403. [CrossRef]

7. Rorat, T.; Szabala, B.M.; Grygorowicz, W.J.; Wojtowicz, B.; Yin, Z.; Rey, P. Expression of sk3-type dehydrin in transporting organs is associated with cold acclimation in solanum species. Planta 2006, 224, 205-221. [CrossRef]

8. Eriksson, S.K.; Kutzer, M.; Procek, J.; Grobner, G.; Harryson, P. Tunable membrane binding of the intrinsically disordered dehydrin Lti30, a cold-induced plant stress protein. Plant Cell 2011, 23, 2391-2404. [CrossRef]

9. Hara, M.; Shinoda, Y.; Kubo, M.; Kashima, D.; Takahashi, I.; Kato, T. Biochemical characterization of the Arabidopsis KS-type dehydrin protein whose gene expression is constitutively abundant rather than stress dependent. Acta Physiol. Plant 2011, 33, 2103-2116. [CrossRef] 
10. Close, T.J. Dehydrins: Emergence of a biochemical role of a family of plant dehydration proteins. Physiol. Plant 1996, 97, 795-803. [CrossRef]

11. Close, T.J. Dehydrins: A commonalty in the response of plants to dehydration and low temperature. Physiol. Plant 1997, 100, 291-296. [CrossRef]

12. Liii, D. Structural motifs in lea proteins. Curr. Top. Plant Physiol. 1993, 10, 91-103.

13. Battaglia, M. The enigmatic lea proteins and other hydrophilins. Plant Physiol. 2008, 148, 6-24. [CrossRef] [PubMed]

14. Dure, L.; Crouch, M.; Harada, J.; Ho, T.H.D.; Mundy, J.; Quatrano, R. Common amino acid sequence domains among the lea proteins of higher plants. Plant Mol. Biol. 1989, 12, 475-486. [CrossRef] [PubMed]

15. Hughes, S.L.; Schart, V.; Malcolmson, J.; Hogarth, K.A.; Martynowicz, D.M.; Tralman-Baker, E. The importance of size and disorder in the cryoprotective effects of dehydrins. Plant Physiol. 2013, 163, 1376-1386. [CrossRef] [PubMed]

16. Nylander, M.; Svensson, J.; Palva, E.T.; Welin, B.V. Stress-induced accumulation and tissue-specific localization of dehydrins in Arabidopsis thaliana. Plant Mol. Biol. 2001, 45, 263-279. [CrossRef]

17. Hundertmar, K.; Hincha, D.K. LEA (Late Embryogenesis Abundant) proteins and their encoding genes in Arabidopsis thaliana. BMC Genomics 2008, 9, 118. [CrossRef] [PubMed]

18. Hara, M.; Shinoda, Y.; Tanaka, Y.; Kuboi, T. DNA binding of citrus dehydrin promoted by zinc ion. Plant Cell Environ. 2009, 32, 532-541. [CrossRef]

19. Koag, M.C. The binding of Maize DHN1 to lipid vesicles. Gain of structure and lipid specificity. Plant Physiol. 2003, 131, 309-316. [CrossRef]

20. Lin, C.H.; Peng, P.H.; Ko, C.Y.; Markhart, A.H.; Lin, T.Y. Characterization of a novel $\mathrm{Y}_{2}$ K-type dehydrin VrDhn1 from Vigna radiata. Plant Cell Physiol. 2012, 53, 930-942. [CrossRef]

21. Rorat, T.; Grygorowicz, W.J.; Irzykowski, W.; Rey, P. Expression of KS-type dehydrins is primarily regulated by factors related to organ type and leaf developmental stage during vegetative growth. Planta 2004, 218, 878-885. [CrossRef] [PubMed]

22. Takebayashi, N.; Brewer, P.B.; Newbigin, E.; Uyenoyama, M.K. Patterns of variation within self-incompatibility loci. Mol. Biol. Evol. 2003, 20, 1778-1794. [CrossRef] [PubMed]

23. Cao, Y.; Xiang, X.; Geng, M.; You, Q.; Huang, X. Effect of HbDHN1 and HbDHN2 Genes on Abiotic Stress Responses in Arabidopsis. Front. Plant Sci. 2017, 8, 470. [CrossRef] [PubMed]

24. Mundy, J.; Chua, N.H. Abscisic acid and water-stress induce the expression of a novel rice gene. EMBO J. 1988, 7, 2279-2286. [CrossRef] [PubMed]

25. Allagulova, C.R.; Gimalov, F.R.; Shakirova, F.M.; Vakhitov, V.A. The plant dehydrins: structure and putative functions. Biochemistry (Moscow) 2003, 68, 945-951. [CrossRef] [PubMed]

26. Zhou, P.; An, Y.; Wang, Z.; Du, H.; Huang, B. Characterization of gene expression associated with drought avoidance and tolerance traits in a perennial grass species. PLoS ONE 2014, 9, e103611. [CrossRef] [PubMed]

27. Hara, M.; Terashima, S.; Fukaya, T.; Kuboi, T. Enhancement of cold tolerance and inhibition of lipid peroxidation by citrus dehydrin in transgenic tobacco. Planta 2003, 217, 290-298. [CrossRef]

28. Shekhawat, U.K.S.; Srinivas, L.; Ganapathi, T.R. MusaDHN-1, a novel multiple stress-inducible SK3-type dehydrin gene, contributes affirmatively to drought- and salt-stress tolerance in banana. Planta 2011, 234, 915-932. [CrossRef]

29. Qin, Y.X.; Qin, F. Dehydrins from wheat $x$ Thinopyrum ponticum amphiploid increase salinity and drought tolerance under their own inducible promoters without growth retardation. Plant Physiol. Biochem. 2016, 99, 142-149. [CrossRef]

30. Danyluk, J.; Perron, A.; Houde, M.; Limin, A.; Fowler, B.; Benhamou, N.; Sarhan, F. Accumulation of an acidic dehydrin in the vicinity of the plasma membrane during cold acclimation of wheat. Plant Cell 1998, 10, 623-638. [CrossRef]

31. Halder, T.; Agarwal, T.; Ray, S. Isolation, cloning, and characterization of a novel Sorghum dehydrin (SbDhn2) protein. Protoplasma 2016, 253, 1475-1488. [CrossRef] [PubMed]

32. Agarwal, T.; Upadhyaya, G.; Halder, T.; Mukherjee, A.; Majumder, A.L.; Ray, S. Different dehydrins perform separate functions in Physcomitrella patens. Planta 2017, 245, 101-118. [CrossRef] [PubMed]

33. Moore, K.; Roberts, L.J. Measurement of lipid peroxidation. Free Radic. Res. 1998, 28, 659-671. [CrossRef] [PubMed] 
34. Bajji, M.; Kinet, J.M.; Lutts, S. The use of the electrolyte leakage method for assessing cell membrane stability as a water stress tolerance test in durum wheat. Plant Growth Regul. 2002, 36, 61-70. [CrossRef]

35. Chen, R.G.; Jing, H.; Guo, W.L.; Wang, S.B.; Ma, F.; Pan, B.G.; Gong, Z.H. Silencing of dehydrin CaDHN1 diminishes tolerance to multiple abiotic stresses in Capsicum annuum L. Plant Cell Rep. 2015, 34, 2189-2200. [CrossRef]

36. Xing, X.; Liu, Y.; Kong, X.; Liu, Y.; Li, D. Overexpression of a maize dehydrin gene, ZmDHN2b, in tobacco enhances tolerance to low temperature. Plant Growth Regul. 2011, 65, 109-118. [CrossRef]

37. Houde, M.; Dallaire, S.N.; Dong, D.; Sarhan, F. Overexpression of the acidic dehydrin WCOR410 improves freezing tolerance in transgenic strawberry leaves. Plant Biotechnol. J. 2004, 2, 381-387. [CrossRef]

38. Hossain, M.A. Hydrogen peroxide priming modulates abiotic oxidative stress tolerance: insights from ROS detoxification and scavenging. Front. Plant Sci. 2015, 6, 420. [CrossRef]

39. Fujita, M.; Fujita, Y.; Maruyama, K.; Seki, M.; Hiratsu, K.; Ohme-Takagi, M.; Tran, L.P.; Yamaguchi-Shinozaki, K.; Shinozaki, K. A dehydration-induced NAC protein, RD26, is involved in a novel ABA-dependent stress-signaling pathway. Plant J. 2004, 39, 863-876. [CrossRef]

40. Biłas, R.; Szafran, K.; Hnatuszko-Konka, K.; Kononowicz, A.K. Cis regulatory elements used to control gene expression in plants. Plant Cell Tissue Organ C. 2016, 127, 269-287. [CrossRef]

41. Lee, S.C.; Kim, S.H.; Kim, S.R. Drought inducible OsDHN1 promoter is activated by OsDREB1A and OsDREB1D. J. Plant Biol. 2013, 56, 115-121. [CrossRef]

42. Zhu, W.N.; Zhang, D.P.; Lu, X.X.; Zhang, L.S.; Yu, Z.Y.; Lv, H. Characterisation of an SKn-type dehydrin promoter from wheat and its responsiveness to various abiotic and biotic stresses. Plant Mol. Biol. Rep. 2014, 32, 664-678. [CrossRef]

43. Lee, S.C.; Lee, M.Y.; Kim, S.J.; Jun, S.H.; An, G.; Kim, S.R. Characterisation of an abiotic stress-inducible dehydrin gene, OsDhn1, in rice (Oryza sativa L.). Mol. Cells 2005, 19, 212-218. [PubMed]

44. Msanne, J.; Lin, J.S.; Stone, J.M.; Awada, T. Characterization of abiotic stress-responsive Arabidopsis thaliana RD29A and RD29B genes and evaluation of transgenes. Planta 2011, 234, 97-107. [CrossRef]

45. Sakuma, Y.; Maruyama, K.; Osakabe, Y.; Qin, F.; Seki, M.; Shinozaki, K.; YamaguchiShinozaki, K. Functional analysis of an Arabidopsis transcription factor, DREB2A, involved in drought-responsive gene expression. Plant Cell 2006, 18, 1292-1309. [CrossRef]

46. Huang, R.W.; Liu, D.F.; Huang, M.; Ma, J.; Li, Z.M.; Li, M.Y.; Sui, S.Z. CpWRKY71, a WRKY Transcription Factor Gene of Wintersweet (Chimonanthus praecox), Promotes Flowering and Leaf Senescence in Arabidopsis. Int. J. Mol. Sci. 2019, 20, 5325. [CrossRef]

47. Guan, H.; Liu, X.; Niu, F.; Zhao, Q.; Fan, N.; Cao, D.; Meng, D.; He, W.; Guo, B.; Wei, Y.; et al. OoNAC72, a NAC-Type Oxytropis ochrocephala Transcription Factor, Conferring Enhanced Drought and Salt Stress Tolerance in Arabidopsis. Front. Plant Sci. 2019, 10, 890. [CrossRef]

48. Shi, X.P.; Ren, J.J.; Yu, Q.; Zhou, S.M.; Ren, Q.P.; Kong, L.J.; Wang, X.L. Overexpression of SDH confers tolerance to salt and osmotic stress, but decreases ABA sensitivity in Arabidopsis. Plant Biol. 2018, 20, 327-337. [CrossRef]

49. Sah, S.K.; Reddy, K.R.; Li, J.X. Abscisic acid and abiotic stress tolerance in crop plants. Front. Plant Sci. 2016, 7, 571. [CrossRef]

50. Zhang, J.H.; Jia, W.S.; Yang, J.C.; Ismail, A.M. Role of ABA in integrating plant responses to drought and salt stresses. Field Crops Res. 2006, 97, 111-119. [CrossRef]

51. Hu, H.H.; You, J.; Fang, Y.J.; Zhu, X.Y.; Qi, Z.Y.; Xiong, L. Characterization of transcription factor gene SNAC2 conferring cold and salt tolerance in rice. Plant Mol. Biol. 2008, 67, 169-181. [CrossRef] [PubMed]

52. Aubert, Y.; Vile, D.; Pervent, M.; Aldon, D.; Ranty, B.; Simonneau, T. RD20, a stress-inducible caleosin, participates in stomatal control, transpiration and drought tolerance in Arabidopsis thaliana. Plant Cell Physiol. 2010, 51, 1975-1987. [CrossRef] [PubMed]

53. Zhai, Y.F.; Wang, H.; Liang, M.M.; Lu, M.H. Both silencing- and over-expression of pepper CaATG8c gene compromise plant tolerance to heat and salt stress. Environ. Exp. Bot. 2017, 141, 10-18. [CrossRef]

54. Koom, J.M.; Matzke, M.A.; Meyer, P. Listening to the silent genes: Transgene silencing, gene regulation and pathogen contro1. Trends Plant Sci. 1999, 4, 340-347.

55. Elmayan, T.; Proux, F.; Vaucheret, H. Arabidopsis RPA2: A genetic link among transcriptional gene silencing, DNA repair, and DNA replication. Curr. Biol. 2005, 15, 1919-1925. [CrossRef] 
56. Chen, R.G.; Guo, W.L.; Yin, Y.X.; Gong, Z.H. A novel F-Box protein CaF-Box is involved in responses to plant hormones and abiotic stress in pepper (Capsicum annuum L.). Int. J. Mol. Sci. 2014, 15, 2413-2430. [CrossRef]

57. Livak, K.J.; Schmittgen, T.D. Analysis of relative gene expression data using real-time quantitative PCR and the 2-11CT method. Methods 2001, 25, 402-408. [CrossRef]

58. Guo, W.L.; Chen, R.G.; Gong, Z.H.; Yin, Y.X.; Li, D.W. Suppression subtractive hybridization analysis of genes regulated by application of exogenous abscisic acid in pepper plant (Capsicum annuum L.) leaves under chilling stress. PLoS ONE 2013, 8, e66667. [CrossRef]

59. Dhindsa, R.S.; Dhindsa, P.P.; Thorpe, T.A. Leaf senescence: Correlated with increased levels of membrane permeability and lipid p. J. Exp. Bot. 1981, 32, 93-101. [CrossRef]

60. Dionisio-Sese, M.L.; Tobita, S. Antioxidant responses of rice seedlings to salinity stress. Plant Sci. 1998, 135, 1-9. [CrossRef]

61. Arkus, K.A.J.; Cahoon, E.B.; Jez, J.M. Mechanistic analysis of wheat chlorophyllase. Arch. Biochem. Biophys. 2005, 438, 146-155. [CrossRef] [PubMed]

62. Liang, X.Q.; Ma, N.N.; Wang, G.D.; Meng, X.; Ai, X.Z.; Meng, Q.W. Suppression of SINAC1 reduces heat resistance in tomato plants. Biol. Plant. 2015, 59, 92-98. [CrossRef]

63. Mittova, V.O.; Volokita, M.; Guy, M.; Tal, M. Activities of SOD and the ascorbate-glutathione cycle enzymes in subcellular compartments in leaves and roots of the cultivated tomato and its wild salt-tolerant relative Lycopersicon pennellii. Plant Physiol. 2000, 110, 42-51. [CrossRef]

64. Brennan, T.; Frenkel, C. Involvement of hydrogen peroxide in the regulation of senescence in pear. Plant Physiol. 1977, 59, 411-416. [CrossRef] [PubMed]

65. Thordal-Christensen, H.; Zhang, Z.; Wei, Y.; Collinge, D.B. Subcellular localization of $\mathrm{H}_{2} \mathrm{O}_{2}$ in plants, $\mathrm{H}_{2} \mathrm{O}_{2}$ accumulation in papillae and hypersensitive response during barley-powdery mildew interaction. Plant J. 1997, 11, 1187-1194. [CrossRef]

66. Jabs, T.; Dietrich, R.A.; Dangl, J.L. Initiation of runaway cell death in an Arabidopsis mutant by extracellular superoxide. Science 1996, 273, 1853-1856. [CrossRef]

67. Wei, W.; Cui, M.Y.; Hu, Y.; Gao, K.; Xie, Y.G.; Jiang, Y.; Feng, J.Y. Ectopic expression of FvWRKY42, a WRKY transcription factor from the diploid woodland strawberry (Fragaria vesca), enhances resistance to powdery mildew, improves osmotic stress resistance, and increases abscisic acid sensitivity in Arabidopsis. Plant Sci. 2018, 275, 60-74. [CrossRef] 